\title{
ON THE STATIC AND DYNAMIC POINTS OF VIEW FOR CERTAIN RANDOM WALKS IN RANDOM ENVIRONMENT *
}

\author{
ERWIN BOLTHAUSEN ${ }^{\dagger}$ AND ALAIN-SOL SZNITMAN ${ }^{\ddagger}$
}

\begin{abstract}
In this work we prove the equivalence between static and dynamic points of views for certain ballistic random walks in random environment on $\mathbb{Z}^{d}$, when $d \geq 4$ and the disorder is low. Our techniques also enable us to derive in the same setting a functional central limit theorem for almost every realization of the environment. We also provide an example where the equivalence between static and dynamic points of views breaks down.
\end{abstract}

0. Introduction. In many models of random motions in random media, the "environment viewed from the particle" naturally defines a Markov chain. The existence of an invariant measure of this chain, which is absolutely continuous with respect to the "static" distribution of the environment is an important property. It is the starting point in the analysis of the "environment viewed from the particle", a technique which has been one of the key tools on the investigation of random motions in random media, c.f. Kipnis-Varadhan [10], S.M. Kozlov [11], Molchanov [13], Olla [14], Papanicolaou-Varadhan [15]. However this technique has had relatively little impact for one of the basic examples of random motions in random media, namely random walks in random environment. In particular, the question of the equivalence between the "static" and "dynamic" distributions of the environment is poorly understood in this situation, with the few exceptions of dimension one, cf. Kesten [9], Molchanov [13], p. 273-274, and of walks with null local drift, cf. Lawler [12], and Papanicolaou-Varadhan [15] in the continuous setting.

The present work proves the equivalence between static and dynamic distributions of the environment, for certain ballistic random walks in random environment, in dimension $d \geq 4$, when the disorder is low. The techniques we develop enable us to derive a "quenched" central limit theorem, which complements the results of Sznitman [18].

Let us now recall the model. The environment in which the walk evolves is described by a collection of i.i.d. (2d)-dimensional vectors, which specify the transition probability of the walk at each site of $\mathbb{Z}^{d}$. We assume that for some $\kappa \in\left[0, \frac{1}{2 d}\right]$,

the common law $\mu$ of the vectors is supported by $\mathcal{P}_{\kappa}$, the set of $(2 d)$-vectors $(p(e))_{|e|=1, e \in \mathbb{Z}^{d}}$, with $p(e) \in[\kappa, 1]$, for $|e|=1$, and $\sum_{|e|=1} p(e)=1$.

Our principal interest lies in the elliptic situation, when

$$
\kappa>0 \text {. }
$$

However, the discussion of what we nickname "directed walks", will also be useful. It

\footnotetext{
*Received June 28, 2001; accepted for publication May 29, 2002.

${ }^{\dagger}$ Institut für Mathematik, Universität Zürich, Winterthurerstrasse 190, 8057 Zürich, Switzerland (eb@amath.unizh.ch). Research supported by Swiss National Science Foundation contract no. 2055648.98 .

‡Departement Mathematik, ETH-Zentrum, CH-8092 Zürich, Switzerland (sznitman@math.ethz.ch).
} 
corresponds to the case

$$
\kappa=0, \text { and } \mu \text {-a.s., } \sum_{i=1}^{d} p\left(e_{i}\right)=1,
$$

with $\left(e_{i}\right)_{1 \leq i \leq d}$, the canonical basis of $\mathbb{R}^{d}$.

The random environment is an element $\omega=(\omega(x, \cdot))_{x \in \mathbb{Z}^{d}}$ of $\Omega=\mathcal{P}_{\kappa}^{\mathbb{Z}^{d}}$, which is endowed with the product $\sigma$-algebra and the product measure $\mathbb{P}=\mu^{\otimes \mathbb{Z}^{d}}$. The random walk in the random environment $\omega$ is the canonical Markov chain $\left(X_{n}\right)_{n \geq 0}$ on $\left(\mathbb{Z}^{d}\right)^{\mathbb{N}}$, with state space $\mathbb{Z}^{d}$ and law $P_{x, \omega}$ starting from $x \in \mathbb{Z}^{d}$, under which

$$
\begin{aligned}
& P_{x, \omega}\left[X_{n+1}=X_{n}+e \mid X_{0}, \ldots, X_{n}\right] \stackrel{P_{x, \omega \omega}=}{=} \omega\left(X_{n}, e\right), n \geq 0,|e|=1, \\
& P_{x, \omega}\left[X_{0}=x\right]=1 .
\end{aligned}
$$

One also introduces the laws defined by the semi-direct product of $\mathbb{P}$ with $P_{x, \omega}$ :

$$
P_{x}=\mathbb{P} \times P_{x, \omega}, \text { on } \Omega \times\left(\mathbb{Z}^{d}\right)^{\mathbb{N}}, \text { for } x \in \mathbb{Z}^{d} .
$$

The environment viewed from the particle is

$$
\bar{\omega}_{n}=t_{X_{n}} \omega=\omega\left(X_{n}+\cdot\right), n \geq 0,
$$

where $t_{x}, x \in \mathbb{Z}^{d}$, denotes the canonical shift on $\Omega$. Under $P_{0}$ (resp. $\left.P_{0, \omega}\right),\left(\bar{\omega}_{n}\right)_{n \geq 0}$ is a Markov chain with state space $\Omega$, initial distribution $\mathbb{P}$ (resp. $\left.\delta_{\omega}\right)$, and transition kernel

$$
R f(\omega)=\sum_{|e|=1} \omega(x, e) f \circ t_{e}(\omega), \omega \in \Omega,
$$

for $f: \Omega \rightarrow \mathbb{R}$, bounded measurable.

We are interested in the question:

$$
\text { does there exist an } R \text {-invariant probability which is absolutely }
$$
continuous with respect to $\mathbb{P}$ ?

It is known that under $(0.2)$ there is at most one such probability, further it is necessarily equivalent to $\mathbb{P}$, and yields an ergodic invariant measure for $R$, cf. Kozlov [11], p. 82, or Lecture 1 of [3]. Our main results concern the case of "non-nestling" walks for which (0.2) holds and

$$
\text { for some } \ell \in S^{d-1}, \eta>0, \mathbb{P} \text {-a.s., } d(x, \omega) \cdot \ell \geq \eta \text {, for all } x \in \mathbb{Z}^{d},
$$

provided:

$$
d(x, \omega)=\sum_{|e|=1} \omega(x, e) e, x \in \mathbb{Z}^{d}, \omega \in \Omega,
$$

is the local drift at site $x$ in the environment $\omega$. It follows from Theorem 3.1 of Sznitman-Zerner [20], that under (0.2), (0.9),

the law $\mathbb{P}_{n}$ of $\bar{\omega}_{n}$ under $P_{0}$ converges weakly to a law $\mathbb{Q}$ on $\Omega$, which is $R$-invariant . 
In the above statement $\Omega$ is endowed with the canonical product topology, for which it is a compact space. We show in Theorem 2.4 that

$$
\text { when } d \geq 4 \text {, and the "disorder is low", } \mathbb{Q} \ll \mathbb{P} \text {, }
$$

in particular this provides a class of examples where (0.8) has a positive answer. The only other known instances where (0.8) has a positive answer correspond to the case $d=1$, when the walk has non-vanishing limiting velocity, cf. Kesten [9], Molchanov [13], p. 273, or to the case $d$ arbitrary with $d(x, \omega) \equiv 0$, cf. Lawler [12]. At the heart of $(0.12)$ lies the fact that under $(0.2),(0.9)$, when $d \geq 4$ and

$$
\alpha=\sup _{|e|=1} \operatorname{ess} \sup \log \frac{p(e)}{\widetilde{p}(e)},(p(\cdot), \widetilde{p}(\cdot) \text { are independent } \mu \text {-distributed }),
$$

is small enough, then

$$
E_{0} \otimes E_{0}\left[\exp \left\{\alpha \sum_{x \in \mathbb{Z}^{d}} L_{X}(x) \wedge L_{\tilde{X}}(x)\right\}\right]<\infty,
$$

where

$$
L_{X}(x)=\sum_{n \geq 0} 1\left\{X_{n}=x\right\}
$$

and $L_{\tilde{X}}$ is defined analogously with $\tilde{X}$, (an independent copy of $X$ ), cf. Theorem 2.4 and 2.6. Note that in $(0.14), \alpha$ appears in the exponential but influences the $P_{0}$-expectation as well.

In Section 1 we also provide an example in the directed situation (0.3) where (0.11) holds but $\mathbb{Q}$ and $\mathbb{P}$ are mutually singular, and (0.8) has a negative answer, cf. Proposition 1.5. The directed situation is of course easier to investigate than the elliptic case. It has a certain analogy with directed polymers in a random environment, cf. Bolthausen [2], Carmona-Molchanov [4], Sinai [16].

As mentioned above, we also derive under similar assumptions as (0.12) a functional central limit theorem under $P_{0, \omega}$ for $\mathbb{P}$-typical $\omega$. It is known from SznitmanZerner [20], that under (0.2), (0.9),

$$
P_{0} \text {-a.s., } \frac{X_{n}}{n} \rightarrow v \text {, where } v \text { is deterministic and } v \cdot \ell>0,
$$

and from Theorem 4.1 and 2.1 of Sznitman [18], that under $P_{0}$,

$$
\text { the sequence of laws of } B_{\cdot}^{n}=\frac{1}{\sqrt{n}}\left(X_{[\cdot n]}-[\cdot n] v\right) \text { on the Skorohod }
$$
space $D\left(\mathbb{R}_{+}, \mathbb{R}^{d}\right)$ converges weakly to the Wiener measure with a non-degenerate covariance matrix $A$.

We show in Theorem 4.2, that when $d \geq 4$ and $\alpha$ in (0.13) is small enough, one can replace in the above statement $P_{0}$ by $P_{0, \omega}$ for $\mathbb{P}$-a.e. $\omega$. We use a "concentration argument", and show that the variance of $E_{0, \omega}\left[f\left(\widetilde{B}_{.}^{n}\right)\right]$ decays rapidly enough for suitable functionals $f$ and $\widetilde{B}^{n}$. the polygonal interpolation of $B^{n}$.

Let us finally explain how this article is organized. Section 1 provides further notations, recalls facts from [20], [3] and derives some preliminary results. It also presents an example in the directed situation where (0.8) has a negative answer. 
Section 2 is principally devoted to the proof of Theorem 2.4, where $(0.12)$ is proven. As mentioned above, the heart of the matter is to control certain quantities like in (0.14), measuring the intersections of two independent copies of $X_{n}$ under $P_{0}$.

Section 3 shows that the counterexample of Section 1 is in essence unstable, and that the directed situation is somehow smoother than that of directed polymers in a random environment.

Section 4 applies the controls of Section 2 to derive variance estimates and a $\mathbb{P}$-almost sure central limit theorem for $B^{n}$.

Finally the Appendix collects certain quantitative estimates on transition probabilities and Green functions of some the random walks which are used in Sections 2 and 4 .

1. Some preliminaries and a counterexample. In this section we first introduce further notations and recall certain results of [3] and [20]. We then provide some useful description of the law $\mathbb{Q}$ alluded to in (0.11) and describe an example in the directed situation $(0.3)$, where $(0.8)$ has a negative answer.

We begin with some notations. We respectively denote by $|\cdot|$ and $\|\cdot\|$ the Euclidean and $\ell_{1}$-distances on $\mathbb{R}^{d}$. We write $B(w, r)$ for the open Euclidean ball of radius $r$ centered at $w \in \mathbb{R}^{d}$. For $U$ a subset of $\mathbb{Z}^{d},|U|$ stands for the cardinality of $U$ and $\partial U$ for the boundary of $U$ :

$$
\partial U=\left\{x \in \mathbb{Z}^{d} \backslash U, \exists y \in U,|y-x|=1\right\} .
$$

We denote by $\left(\theta_{n}\right)_{n \geq 0}$, the canonical shift on $\left(\mathbb{Z}^{d}\right)^{\mathbb{N}}$, and write $\left(\mathcal{F}_{n}\right)_{n \geq 0}$ for the canonical filtration on $\left(\mathbb{Z}^{d}\right)^{\mathbb{N}}$ attached to the canonical process $\left(X_{n}\right)_{n \geq 0}$. For $U \subseteq \mathbb{Z}^{d}$, we let $H_{U}$ and $T_{U}$ respectively stand for the entrance time in $U$ and exit time from $U$ :

$$
H_{U}=\inf \left\{n \geq 0, X_{n} \in U\right\}, T_{U}=\inf \left\{n \geq 0, X_{n} \notin U\right\} .
$$

When $U=\{y\}$, we tacitly write $H_{y}$ in place of $H_{\{y\}}$. For $\ell \in S^{d-1}, u \in \mathbb{R}$, we shall often encounter

$$
T_{u}^{\ell}=\inf \left\{n \geq 0, X_{n} \cdot \ell \geq u\right\}, \widetilde{T}_{u}^{\ell}=\inf \left\{n \geq 0, X_{n} \cdot \ell \leq u\right\}
$$

as well as the first backtracking time in the direction $\ell$ :

$$
D^{\ell}=\inf \left\{n \geq 0, X_{n} \cdot \ell<X_{0} \cdot \ell\right\} .
$$

When $A$ is an event, $h$ a random variable and $Q$ a probability, we sometimes use the notations $E^{Q}[A, h]$ or $Q(A, h)$ in place of $\int_{A} h d Q$. Further $\mathbb{E}[\cdot]$ always refer to the $\mathrm{P}$-expectation.

We now recall the construction of a renewal structure for random walks in random environment transient in a given direction, following Sznitman-Zerner [20]. We assume that (0.2) holds, $\ell \in S^{d-1}$ is such that

$$
P_{0}\left[\lim _{n} X_{n} \cdot \ell=\infty\right]=1,
$$

and $a$ is a positive number restricted by

$$
0<a \leq 10 \sqrt{d}
$$


(although nothing special happens for $a>10 \sqrt{d}$, this restriction will be convenient to remove the dependence on $a$ of some constants). We introduce two sequences of stopping times, $S_{k}, k \geq 0$, and $R_{k}, k \geq 1$, together with the sequence $M_{k}, k \geq 0$, of successive maxima of the walk in the direction $\ell$ :

$$
\begin{aligned}
S_{0}= & 0, M_{0}=X_{0} \cdot \ell, \\
& \text { and by induction, when } k \geq 0, \text { we set } \\
S_{k+1}= & T_{M_{k}+a} \leq \infty, R_{k+1}=D^{\ell} \circ \theta_{S_{k+1}}+S_{k+1} \leq \infty, \\
M_{k+1}= & \sup \left\{X_{n} \cdot \ell, 0 \leq n \leq R_{k+1}\right\} .
\end{aligned}
$$

Remark that $S_{k+1}<\infty, P_{0}$-a.s. on $\left\{R_{k}<\infty\right\}$. We thus have

$$
0=S_{0} \leq S_{1} \leq R_{1} \leq S_{2} \leq \cdots \leq \infty,
$$

and these inequalities are strict if the left member is finite. We can now introduce:

$$
K=\inf \left\{k \geq 1, S_{k}<\infty, R_{k}=\infty\right\} .
$$

As shown in Proposition 1.2 of Sznitman-Zerner [20], under (0.2), (1.5):

$$
P_{0}\left[D^{\ell}=\infty\right]>0 \text {, and } P_{0} \text {-a.s., } K<\infty .
$$

The key renewal time is now defined via:

$$
\tau_{1}=S_{K},
$$

and inductively for $k \geq 1$,

$$
\tau_{k+1}=\tau_{1}(X)+\tau_{k}\left(X_{\tau_{1}+}-X_{\tau_{1}}\right),\left(\tau_{k+1}=\infty, \text { when } \tau_{k}=\infty\right) .
$$

It is shown in Theorem 1.4 of [20] that

$$
P_{0^{-} \text {a.s., }} 0<\tau_{1}<\tau_{2}<\cdots<\tau_{k}<\ldots,
$$

and one has the renewal property:

under $P_{0},\left(\left(X_{\tau_{1} \wedge}\right), \tau_{1}\right),\left(\left(X_{\left(\tau_{1}+\cdot\right) \wedge \tau_{2}}-X_{\tau_{1}}\right), \tau_{2}-\tau_{1}\right), \ldots,\left(\left(X_{\left(\tau_{k}+\cdot\right) \wedge \tau_{k+1}}-X_{\tau_{k}}\right)\right.$, $\left.\tau_{k+1}-\tau_{k}\right), \ldots$ are independent variables and starting with the second term of this sequence their distribution coincide with the distribution of $\left(\left(X_{\tau_{1} \wedge}\right), \tau_{1}\right)$ under $P_{0}\left[\cdot \mid D^{\ell}=\infty\right]$.

We still need some further notations. For $k \geq 1, y \in \mathbb{Z}^{d}$, we let $A_{k}(y)$ denote the event:

$$
A_{k}(y)=\left\{D^{\ell}>H_{y}, \tau_{k}\left(\bar{X}^{y}\right)=H_{y}\right\},
$$

where $\bar{X}^{y}$. stands for an arbitrary path which coincides with $X$. up to time $H_{y}$ and such that $\bar{X}_{m}^{y} \cdot \ell \geq y \cdot \ell$, for $m>H_{y}$. Note that this unambiguously defines $A_{k}(y)$. We then consider for $k \geq 0, x, y \in \mathbb{Z}^{d}$ :

$$
\begin{aligned}
& q_{k}(x, y, \omega)=\delta_{x, y}, \quad \text { when } k=0 \\
& P_{x, \omega}\left[A_{k}(y)\right], \quad \text { when } k \geq 1 \text {, }
\end{aligned}
$$


of course $q_{k}(x, y, \omega)$ vanishes if $(y-x) \cdot \ell<k a$. The kernels $q_{k}(\cdot, \cdot, \omega)$ satisfy a semigroup property:

\section{LEMMA 1.1.}

$$
q_{k+n}(x, y, \omega)=\sum_{z} q_{k}(x, z, \omega) q_{n}(z, y, \omega), \text { for } k, n \geq 0, x, y \in \mathbb{Z}^{d}, \omega \in \Omega .
$$

Proof. Without loss of generality we assume $k, n \geq 1$, so that in the notations of $(1.13)$

$$
\begin{aligned}
& q_{k+n}(x, y, \omega)=P_{x, \omega}\left[D^{\ell}>H_{y}, \tau_{k+n}\left(\bar{X}^{y}\right)=H_{y}\right] \\
& =\sum_{z} P_{x, \omega}\left[D^{\ell}>H_{y}, X_{\tau_{k}\left(\bar{X}^{y}\right)}=z, \tau_{k+n}\left(\bar{X}^{y}\right)=H_{y}\right] \\
& =\sum_{z} P_{x, \omega}\left[\left\{D^{\ell}>H_{z}, \tau_{k}\left(\bar{X}^{z}\right)=z\right\} \cap \theta_{H_{z}}^{-1}\left(A_{n}(y)\right)\right]=\sum_{z} q_{k}(x, z, \omega) q_{n}(z, y, \omega),
\end{aligned}
$$

using the strong Markov property in the last step.

We now further assume that

$$
E_{0}\left[\tau_{1} \mid D^{\ell}=\infty\right]<\infty
$$

so that from Proposition 2.1 of [20], the law of large numbers, cf. (0.16), follows with

$$
v=\frac{E_{0}\left[X_{\tau_{1}} \mid D^{\ell}=\infty\right]}{E_{0}\left[\tau_{1} \mid D^{\ell}=\infty\right]} .
$$

The assumptions (1.5), (1.16) hold for instance under (0.9), but also in many other situations, cf. Sznitman-Zerner [20] and Sznitman [19]. We are now ready to introduce in greater generality than mentioned in $(0.11)$ a probability $\mathbb{Q}$ which plays an important role in the sequel.

Proposition 1.2. ( $d \geq 1$, under (0.2), (1.5), (1.16))

There exists a probability $\mathbb{Q}_{0}$ on $\Omega$, such that for $N \geq 1$ and $h$ bounded $\sigma(\omega(x, \cdot)$, $\ell \cdot x \geq-N a)$-measurable,

$$
\mathbb{Q}_{0}(h)=\sum_{x \in \mathbb{Z}^{d}} \mathbb{E}\left[h q_{N}(x, 0, \omega) P_{0, \omega}\left[D^{\ell}=\infty\right]\right] / P_{0}\left[D^{\ell}=\infty\right] .
$$

Then $\mathbb{Q}_{0}$-a.s., $P_{0, \omega}\left[D^{\ell}=\infty\right]>0$, and one can define a probability $\mathbb{Q}$ on $\Omega$ such that for $h$ bounded measurable

$$
\mathbb{Q}(h)=\sum_{u \geq 0} \int E_{0, \omega}\left[u<\tau_{1}, h\left(t_{X_{u}} \omega\right) \mid D^{\ell}=\infty\right] d \mathbb{Q}_{0}(\omega) / E_{0}\left[\tau_{1} \mid D^{\ell}=\infty\right] .
$$

If $h$ is bounded and satisfies the same measurability assumption as in (1.18),

$$
\mathbb{Q}(h)=\sum_{u \geq 0} E_{0}\left[\tau_{N} \leq u<\tau_{N+1}, h\left(t_{X_{u}} \omega\right) \mid D^{\ell}=\infty\right] / E_{0}\left[\tau_{1} \mid D^{\ell}=\infty\right] .
$$

Further in the notations of (0.7),

$(1.21) \quad \mathbb{Q}$ is $R$-invariant and the laws of $\bar{\omega}_{n}$ under $P_{0}$ converge weakly towards $\mathbb{Q}$. 
(In particular $\mathbb{Q}$ does not depend on the specific choice of $\ell$ and a for which (1.5), (1.16) hold).

Proof. Denote by $B_{N, h}$ the righthand side of (1.18). Then for $N \geq 1, k \geq 1, h$ as in $(1.18)$,

$$
B_{N+k, h} P_{0}\left[D^{\ell}=\infty\right] \stackrel{(1.15)}{=} \sum_{x, z} \mathbb{E}\left[q_{k}(x, z, \omega) h q_{N}(z, 0, \omega) P_{0, \omega}\left[D^{\ell}=\infty\right]\right]
$$

and non-vanishing summands require that $x \cdot \ell<z \cdot \ell \leq-N a$. Note that $q_{k}(x, z, \omega)$ is $\sigma(\omega(y, \cdot), y \cdot \ell<z \cdot \ell)$-measurable, whereas $h q_{N}(z, 0, \omega) P_{0, \omega}\left[D^{\ell}=\infty\right]$ is $\sigma(\omega(y, \cdot)$, $y \cdot \ell \geq z \cdot \ell)$-measurable. Hence using independence under $\mathbb{P}$,

$$
\begin{aligned}
& B_{N+k, h} P_{0}\left[D^{\ell}=\infty\right]=\sum_{x, z} \mathbb{E}\left[q_{k}(x, z, \omega)\right] \mathbb{E}\left[h q_{N}(z, 0, \omega) P_{0, \omega}\left[D^{\ell}=\infty\right]\right] \\
& \stackrel{\text { translation }}{\text { invariance }}=B_{N, h} P_{0}\left[D^{\ell}=\infty\right] \sum_{y} \mathbb{E}\left[q_{k}(0, y, \omega)\right]
\end{aligned}
$$

and by a similar reasoning as above

$$
\begin{aligned}
& =B_{N, h} \sum_{y} \mathbb{E}\left[q_{k}(0, y, \omega) P_{y, \omega}\left[D^{\ell}=\infty\right]\right] \\
& \stackrel{(1.13)-(1.14)}{=} B_{N, h} \sum_{y} E_{0}\left[D^{\ell}=\infty, X_{\tau_{k}}=y\right]=B_{N, h} P_{0}\left[D^{\ell}=\infty\right] .
\end{aligned}
$$

A similar calculation shows that for $N \geq 1$,

$$
B_{N, 1}=\sum_{x} \mathbb{E}\left[q_{N}(x, 0, \omega) P_{0, \omega}\left[D^{\ell}=\infty\right]\right] / P_{0}\left[D^{\ell}=\infty\right]=1 .
$$

The claim (1.18) now follows from Kolmogorov's extension theorem. Note that choosing $h=1\left\{P_{0, \omega}\left[D^{\ell}=\infty\right]=0\right\}$, we find

$$
\mathbb{Q}_{0} \text {-a.s., } P_{0, \omega}\left[D^{\ell}=\infty\right]>0 .
$$

Note that as a consequence of the spatial ergodicity of $\mathbb{P},(0.2)$, and (1.9), the Markov property shows that

$$
\mathbb{P} \text {-a.s., for all } x \in \mathbb{Z}^{d}, P_{x, \omega}\left[D^{\ell}=\infty\right]>0 \text {. }
$$

The formula (1.19) is well defined, and using the fact that $E_{0, \omega}\left[u<\tau_{1} \mid D^{\ell}=\infty\right]$ is $\sigma(\omega(y, \cdot), y \cdot \ell \geq 0)$-measurable and (1.18), one readily checks as above that $\mathbb{Q}$ is a probability. Let us prove (1.20). For $h$ as in (1.20), $u \geq 0, E_{0, \omega}\left[h\left(t_{X_{u}} \omega\right)\right.$, $\left.u<\tau_{1} \mid D^{\ell}=\infty\right]$ is bounded $\sigma(\omega(y, \cdot), y \cdot \ell \geq-N a)$-measurable, and by (1.18),

$$
\begin{aligned}
& \mathbb{Q}(h) E_{0}\left[\tau_{1}, D^{\ell}=\infty\right]=\sum_{u \geq 0, x} \mathbb{E}\left[q_{N}(x, 0, \omega) E_{0, \omega}\left[h\left(t_{X_{u}} \omega\right), D^{\ell}=\infty, u<\tau_{1}\right]\right] \\
& \stackrel{\text { translation }}{\text { invariance }}=\sum_{u \geq 0, z} \mathbb{E}\left[q_{N}(0, z, \omega) E_{z, \omega}\left[h\left(t_{X_{u}} \omega\right), D^{\ell}=\infty, u<\tau_{1}\right]\right]
\end{aligned}
$$

using now the Markov property together with (1.13), (1.14),

$$
\begin{aligned}
& =\sum_{u \geq 0, z} \mathbb{E}\left[E_{0, \omega}\left[D^{\ell}=\infty, X_{\tau_{N}}=z, u<\tau_{N+1}-\tau_{N}, h\left(t_{X_{u+\tau_{N}}} \omega\right)\right]\right] \\
& =\sum_{s \geq 0} E_{0}\left[\tau_{N} \leq s<\tau_{N+1}, D^{\ell}=\infty, h\left(t_{X_{s}} \omega\right)\right]
\end{aligned}
$$


which proves (1.20).

We now turn to the proof of (1.21). We first show that the laws of $\bar{\omega}_{n}$ under $P_{0}$ converge weakly in Cesaro sense to $\mathbb{Q}$. The argument is essentially a repetition of the proof of Theorem 3.1 of Sznitman-Zerner [20], (see below (3.6)). The only variation (cf. below (3.7) of [20]), is that one uses here that for $u \geq 0$,

(1.28) $\frac{1}{M} \sum_{n=0}^{M-1} P_{0}\left[n-u=\tau_{k}\right.$, for some $\left.k \geq 1\right] \longrightarrow 1 / E_{0}\left[\tau_{1} \mid D^{\ell}=\infty\right]$, as $M \rightarrow \infty$,

as easily follows from the renewal property (1.12).

We hence see that (1.19) defines a unique probability $\mathbb{Q}$ regardless of the specific choice of $\ell$ and $a$ for which (1.5), (1.16) hold. Observe that if we choose $a^{\prime} \in(0, a]$, such that

$$
a^{\prime}<\inf \{\ell \cdot e ; \ell \cdot e>0,|e|=1\},
$$

then $P_{0}$-a.s. the corresponding variable $\tau_{1}^{\prime}$ will be smaller than $\tau_{1}$ corresponding to $a$, and (1.16) will hold for $\tau_{1}^{\prime}$ as well. In other words we can assume that $a<\inf \{\ell \cdot e$; $\ell \cdot e>0,|e|=1\}$, and now the proof of Theorem 3.1 of [20] applies and shows that the laws of $\bar{\omega}_{n}$ under $P_{0}$ converge weakly to $\mathbb{Q}$. Since $R$ preserves the set of bounded continuous functions on $\omega$, the $R$-invariance of $\mathbb{Q}$ follows immediately as in the proof of Theorem 3.1 of [20]. This finishes the proof of Proposition 1.2.

The next lemma will be helpful in the next section.

Lemma 1.3. ( $d \geq 1$, under $(0.2),(1.5),(1.16))$

$$
\mathbb{Q} \ll \mathbb{P} \text { if and only if } \mathbb{Q}_{0} \ll \mathbb{P} \text {. }
$$

Proof. Inspecting the term corresponding to $u=0$ in (1.19), we see that $\mathbb{Q}_{0} \leq$ $\mathbb{E}_{0}\left[\tau_{1} \mid D^{\ell}=\infty\right] \mathbb{Q}$, and hence if $\mathbb{Q} \ll \mathbb{P}, \mathbb{Q}_{0} \ll \mathbb{P}$ follows. Conversely if $\mathbb{Q}_{0} \ll \mathbb{P}$, from (1.19) for any measurable subset $A$ of $\Omega$,

$$
E_{0}\left[\tau_{1} \mid D^{\ell}=\infty\right] \mathbb{Q}(A)=\sum_{u \geq 0, y} \int 1_{A} \circ t_{y} P_{0, \omega}\left[u<\tau_{1}, X_{u}=y \mid D^{\ell}=\infty\right] d \mathbb{Q}_{0}(\omega),
$$

and hence if $\mathbb{P}(A)=0, \mathbb{Q}(A)=0$ follows. As a result $\mathbb{Q} \ll \mathbb{P}$.

For later use we introduce the notation

$$
f_{N}(\omega)=\sum_{x} q_{N}(x, 0, \omega), \text { for } N \geq 1, \omega \in \Omega,
$$

in particular in view of (1.18), for $N \geq 1$,

$$
\text { on } \sigma(\omega(x, \cdot), \ell \cdot x \geq-N a), \mathbb{Q}_{0} \text { coincides with } f_{N} \frac{P_{0, \omega}\left[D^{\ell}=\infty\right]}{P_{0}\left[D^{\ell}=\infty\right]} \mathbb{P} \text {. }
$$

We now turn to the directed situation and assume (0.3) for the remainder of this section. We shall in particular describe an example where (0.8) has a negative answer. It is convenient to introduce the following compact subset of $\Omega$ which has full $\mathbb{P}$ measure:

$$
\Omega_{\mathrm{dir}}=\left\{\omega \in \mathcal{P}_{0}^{\mathbb{Z}^{d}}=\Omega, \text { for all } x \in \mathbb{Z}^{d}, \sum_{i=1}^{d} \omega\left(x, e_{i}\right)=1\right\}
$$


By analogy with (1.14), (1.30), we introduce

$$
\begin{aligned}
p_{k}(x, y, \omega) & =P_{x, \omega}\left[X_{k}=y\right], x, y \in \mathbb{Z}^{d}, k \geq 0, \omega \in \Omega_{\mathrm{dir}}, \\
g_{N}(\omega) & =\sum_{x} p_{N}(x, 0, \omega), N \geq 0, \omega \in \Omega_{\text {dir }} .
\end{aligned}
$$

We also introduce on $\Omega_{\text {dir }}$ the filtration:

$$
\mathcal{H}_{N}=\sigma\left(\omega(x, \cdot), x_{1}+\cdots+x_{d} \geq-N\right), N \geq 0
$$

Proposition 1.4 .

$g_{N}$ is an $\mathcal{H}_{N}$-martingale under $\mathbb{P}$.

There is a unique probability $\mathbb{Q}$ on $\Omega_{\mathrm{dir}}$ such that for $N \geq 0$, the restriction of $\mathbb{Q}$ to $\mathcal{H}_{N}$ coincides with $g_{N} \mathbb{P}$.

The law of $\bar{\omega}_{N}$ under $P_{0}$ is $g_{N} \mathbb{P}$ and it converges weakly to $\mathbb{Q}$ which is $R$-invariant.

Proof. The claim (1.37) is an immediate consequence of (1.36) and the fact that $g_{0}=1$. Let us prove (1.36). For $\omega \in \Omega_{\text {dir }}$, a summand in (1.34) vanishes when $x_{1}+\cdots+x_{d} \neq-N$. The fact that $g_{N}$ is $\mathcal{H}_{N}$-measurable easily follows. Further, for $N \geq 0$, using the Markov property,

$$
\begin{aligned}
\mathbb{E}\left[g_{N+1} \mid \mathcal{H}_{N}\right] & =\sum_{x, y} p_{N}(x, 0, \omega) \mathbb{E}\left[p_{1}(y, x, \omega) \mid \mathcal{H}_{N}\right] \\
& =\sum_{x} p_{N}(x, 0, \omega) \sum_{i=1}^{d} \mathbb{E}\left[p_{1}\left(-e_{i}+x, x, \omega\right) \mid \mathcal{H}_{N}\right]
\end{aligned}
$$

using independence and translation invariance, we find

$$
=\sum_{x} p_{N}(x, 0, \omega) \sum_{i=1}^{d} \mathbb{E}\left[p_{1}\left(0, e_{i}, \omega\right)\right]=g_{N},
$$

which finishes the proof of (1.36).

Let us prove (1.38). For bounded measurable $h$

$$
E_{0}\left[h\left(\bar{\omega}_{N}\right)\right]=\sum_{y} \mathbb{E}\left[h \circ t_{y} P_{0, \omega}\left[X_{N}=y\right]\right]
$$

using translation invariance and the notation (1.33),

$$
=\sum_{y} \mathbb{E}\left[h p_{N}(-y, 0, \omega)\right]=\mathbb{E}\left[h g_{N}\right] .
$$

From (1.36), we immediately conclude that the laws of $\bar{\omega}_{N}$ under $P_{0}$ converge weakly to $\mathbb{Q}$. The $R$-invariance of $\mathbb{Q}$ follows as in the proof of Proposition 1.2.

We shall now see that the martingale $g_{N}$ need not be uniformly integrable and this will lead to a law $\mathbb{Q}$ which is not absolutely continuous with respect to $\mathbb{P}$. We now specify the law of the environment at one site via:

$$
\mathbb{P}\left[\omega\left(0, e_{i}\right)=1\right]=\frac{1}{d}, \quad i=1, \ldots, d .
$$


In other words, the environment is obtained by picking at each site in an i.i.d. and uniform fashion, one of the vectors of the canonical basis of $\mathbb{R}^{d}$. Once the environment is chosen the walk moves deterministically, following at each step the direction chosen at the site where it stands.

Proposition 1.5. ( $d \geq 2$, under (1.41))

$$
\mathbb{Q} \perp \mathbb{P}
$$

(1.43) There is no R-invariant probability absolutely continuous with respect to $\mathbb{P}$.

Proof. We first prove (1.42). From (1.34), (1.41) we know that $g_{N}$ is a martingale with values in $\mathbb{N}$. It converges $\mathbb{P}$-a.s. to the $\mathbb{N}$-valued variable $g_{\infty}$. We shall now prove that:

$$
\text { P-a.s., } g_{\infty}=0 \text {. }
$$

The claim (1.42) will follow at once since from (1.44)

$$
\mathbb{P}\left[\bigcap_{N \geq 0}\left\{g_{N} \geq 1\right\}\right]=0, \text { whereas } \mathbb{Q}\left(\bigcap_{N \geq 0}\left\{g_{N} \geq 1\right\}\right)=1 .
$$

To prove (1.44) observe that when $U$ is a finite non-empty subset of $\left\{x: x_{1}+\cdots+x_{d}=\right.$ $-N\}$, one can find $y \in U$ with $y+e_{2}-e_{1} \notin U$. Hence $y-e_{1}+e_{1} \in U$, but $y-e_{1}+e_{2} \notin U$. Further the set $\bigcup_{i=1}^{d}\left(U-e_{i}\right) \subseteq\left\{x: x_{1}+\cdots+x_{d}=-N-1\right\}$ has at most cardinality $d|U|$, and we thus see that for $N \geq 0$, $\mathbb{P}$-a.s. on $\left\{U=\left\{x \in \mathbb{Z}^{d}, p_{N}(x, 0, \omega)=1\right\}\right\} \in$ $\mathcal{H}_{N}, \mathbb{P}\left[g_{N+1} \neq g_{N} \mid \mathcal{H}_{N}\right] \geq\left(\frac{1}{d}\right)^{d|U|}$.

Therefore for $n \geq 0, K \geq 1, \mathbb{P}$-a.s. on $\left\{g_{N}=K\right\}$,

$$
\mathbb{P}\left[g_{N+1} \neq g_{N} \mid \mathcal{H}_{N}\right] \geq d^{-d K} .
$$

It then follows from Borel-Cantelli's lemma, cf. Durrett [6], p. 207-208, that $\mathbb{P}$-a.s. on the event $\lim \inf \left\{g_{N}=K\right\}, g_{N+1} \neq g_{N}$ for infinitely many $N$. Since $\mathbb{P}$-a.s., $g_{N}$ converges to the integer-valued $g_{\infty}$, we see that (1.44) holds.

We now turn to the proof of (1.43). Let $\widetilde{\mathbb{Q}} \ll \mathbb{P}$ be an $R$-invariant probability, then for $N \geq 1$ :

$$
\int 1\left\{g_{N} \geq 1\right\} d \widetilde{\mathbb{Q}}=\int R^{N} 1\left\{g_{N} \geq 1\right\} d \widetilde{\mathbb{Q}} .
$$

Observe that $\mathbb{P}$-a.s.,

$$
\begin{aligned}
R^{N} 1\left\{g_{N} \geq 1\right\} & =\sum_{y} p_{N}(0, y, \omega) 1\left\{\sum_{x} p_{N}\left(x, 0, t_{y} \omega\right) \geq 1\right\} \\
& =\sum_{y} p_{N}(0, y, \omega) 1\left\{\sum_{x} p_{N}(x, y, \omega) \geq 1\right\}=1 .
\end{aligned}
$$

As a result, for $N \geq 1$,

$$
1=\widetilde{\mathbb{Q}}\left(g_{N} \geq 1\right)=\widetilde{\mathbb{Q}}\left(\bigcap_{N \geq 1}\left\{g_{N} \geq 1\right\}\right),
$$

and in view of the first equality of (1.45), this implies $\widetilde{\mathbb{Q}} \perp \mathbb{P}$, a contradiction.

It is natural to wonder whether the above example of negative answer to (0.8) persists when one considers directed walks which jump according to small random perturbations of a direction prescribed at each site by the environment. We shall return to this question in Section 3. 
2. Static and dynamic points of views for non-nestling walks. Throughout this section, we assume $(0.2)$. The main object is to prove that in the non-nestling case, when $d \geq 4$ and the disorder is low (i.e. $\alpha$ in (0.13) is small), the $R$-invariant measure $\mathbb{Q}$ of Proposition 1.2 is absolutely continuous with respect to $\mathbb{P}$, and hence (0.8) has a positive answer.

Our strategy is to show that $\sup _{N} \mathbb{E}\left[f_{N}^{2}\right]<\infty$, to deduce that $\mathbb{Q}_{0} \ll \mathbb{P}$, cf. (1.30), and conclude that $\mathbb{Q} \ll \mathbb{P}$ with the help of Lemma 1.3. For the time being we assume (0.2) and $\ell \in S^{d-1}$ is such that (1.5) holds, that is:

$$
P_{0}\left[\lim X_{n} \cdot \ell=\infty\right]=1 .
$$

We choose some $a \in(0,10 \sqrt{d}]$, and consider the variables $\tau_{k}, k \geq 1$, of $(1.10),(1.11)$. It is convenient to introduce the set $I$ of increasing integer-valued functions on $\mathbb{N}$, with value 0 at 0 , as well as the product space $\sum=I \times\left(\mathbb{Z}^{d}\right)^{\mathbb{N}}$, which is endowed with the canonical product $\sigma$-algebra and the canonical processes $\left(s_{i}\right)_{i \geq 0},\left(Y_{n}\right)_{n \geq 0}$. We consider the law $Q$ on $\sum$ under which:

$$
\begin{aligned}
& \left(s_{i}, Y_{s_{i}}\right)_{i \geq 0} \text { is a random walk on } \mathbb{N} \times \mathbb{Z}^{d} \text { starting in }(0,0), \text { with jump } \\
& \text { distribution } Q\left(s_{1}=s, Y_{s_{1}}=y\right)=P_{0}\left[X_{\tau_{1}}=-y, \tau_{1}=s \mid D^{\ell}=\infty\right]= \\
& P_{y}\left[X_{\tau_{1}}=0, \tau_{1}=s \mid D^{\ell}=\infty\right]
\end{aligned}
$$

and conditionally on $\left(s_{i}, Y_{s_{i}}\right)_{i \geq 0}$, the law of $Y$. under $Q$ is obtained by gluing together independent bridges which interpolate between positions $Y_{s_{i}}$ at times $s_{i}$ and position $Y_{s_{i+1}}$ at times $s_{i+1}$ and are modelled on $P_{Y_{s_{i+1}}}\left[X_{\tau_{1}+s_{i}-} \in \cdot \mid D^{\ell}=\infty, X_{\tau_{1}}=Y_{s_{i}}\right.$, $\left.\tau_{1}=s_{i+1}-s_{i}\right]$ :

$$
\begin{aligned}
& Q\left(\left(Y_{s_{i}+\wedge}\left(s_{i+1}-s_{i}\right)\right.\right. \\
& )_{i \geq 0} \in \cdot \mid\left(s_{i}, Y_{s_{i}}\right)_{i \geq 0}\right)= \\
& \otimes_{i \geq 0} P_{Y_{s_{i+1}}}\left[X_{\left(\tau_{1}-\cdot\right)_{+}} \in \cdot \mid D^{\ell}=\infty, X_{\tau_{1}}=Y_{s_{i}}, \tau_{1}=s_{i+1}-s_{i}\right] .
\end{aligned}
$$

For $y \in \mathbb{Z}^{d}$, we denote by $Q_{y}$ the law on $\sum$ of $\left(s_{i}\right),\left(y+Y_{n}\right)$. Further for $y, \widetilde{y} \in \mathbb{Z}^{d}, Q_{y}, \widetilde{y}$ stands for the product measure $Q_{y} \otimes Q_{\widetilde{y}}$ on $\sum \times \sum$. Also in what follows processes referring to the second component of the product space are denoted with a superscript $\sim$, such as for instance $\left(\widetilde{s}_{i}\right)$ or $(\widetilde{Y}$.). We now provide a more tractable upper bound on $\mathbb{E}\left[f_{N}^{2}\right]$, where the coefficient $\alpha$ of $(0.13)$ and expressions similar to (0.14) come into play.

LEMMA 2.1. (under (0.2), (1.5), (1.6))

$$
\mathbb{E}\left[f_{N}^{2}\right] \leq Q_{0,0}\left[\exp \left\{\alpha \sum_{x} L_{N}(x) \wedge \widetilde{L}_{N}(x)\right\}\right], \text { for } N \geq 1
$$

where for $N \geq 1, x \in \mathbb{Z}^{d}$ :

$$
L_{N}(x)=\sum_{0<n \leq s_{N}} 1\left\{Y_{n}=x\right\}
$$

Proof. For $N \geq 1$, we consider the collection of finite length nearest neighbor paths on $\mathbb{Z}^{d}$ :

$$
\begin{aligned}
\mathcal{P}_{N}= & \left\{(\sigma(n))_{n \in[0, m]}: m \geq N, \sigma(n) \in \mathbb{Z}^{d}, \sigma(n) \cdot \ell \geq \sigma(0) \cdot \ell, \text { for } 0 \leq n \leq m,\right. \\
& \left.|\sigma(n+1)-\sigma(n)|=1 \text { for } 0 \leq n<m, \sigma(m)=0, \text { and } \tau_{N}\left(\overline{\sigma(\cdot)}^{0}\right)=m\right\},
\end{aligned}
$$


where just as below (1.13) $\overline{\sigma(\cdot)}^{0}$ stands for an arbitrary path coinciding with $\sigma(\cdot)$ up to its entrance time in 0 , which afterward remains in $\{z: z \cdot \ell \geq 0\}$. For $\sigma \in \mathcal{P}_{N}$, we denote by $\{X \succeq \sigma\}$ the event $\left\{X_{n}=\sigma(n), 0 \leq n \leq m\right\}$, with $m$ the terminal time of $\sigma$. From (1.30), (1.13), (1.14), we find for $N \geq 1$,

$$
\begin{aligned}
f_{N} & =\sum_{x} P_{x, \omega}\left[D^{\ell}>H_{0}, \tau_{N}\left(\bar{X}^{0}\right)=H_{0}\right]=\sum_{\sigma \in \mathcal{P}_{N}} P_{\sigma(0), \omega}[X \succeq \sigma] \\
& =\sum_{\sigma \in \mathcal{P}_{N}} \prod_{n=0}^{m-1} \omega(\sigma(n), \sigma(n+1)-\sigma(n))=\sum_{\sigma \in \mathcal{P}_{N}} \prod_{x \in \mathbb{Z}^{d}} \omega(x, e)^{L_{\sigma}(x, e)}
\end{aligned}
$$

where for $\sigma \in \mathcal{P}_{N}, x \in \mathbb{Z}^{d},|e|=1$,

$$
L_{\sigma}(x, e)=\sum_{n=0}^{m-1} 1\{\sigma(n)=x, \sigma(n+1)=x+e\}, L_{\sigma}(x)=\sum_{|e|=1} L_{\sigma}(x, e) .
$$

Hence with hopefully obvious notations, for $N \geq 1$ :

$$
\begin{aligned}
& \mathbb{E}\left[f_{N}^{2}\right]=\sum_{\sigma, \tilde{\sigma} \in \mathcal{P}_{N}} \mathbb{E}\left[\prod_{\substack{x \in \mathbb{Z}^{d} \\
|e|=1}} \omega(x, e)^{L_{\sigma}(x, e)+L_{\tilde{\sigma}}(x, e)}\right] \\
& \stackrel{\text { independence }}{=} \sum_{\sigma, \widetilde{\sigma} \in \mathcal{P}_{N}} \prod_{x \in \mathbb{Z}^{d}} \mathbb{E}\left[\prod_{|e|=1} \omega(x, e)^{L_{\sigma}(x, e)+L_{\tilde{\sigma}}(x, e)}\right] .
\end{aligned}
$$

Note that for any $n(e), \widetilde{n}(e) \geq 0,|e|=1$, and $n=\sum_{e} n(e), \widetilde{n}=\sum_{e} \widetilde{n}(e)$,

$$
\mathbb{E}\left[\prod_{|e|=1} \omega(0, e)^{n(e)+\widetilde{n}(e)}\right] \stackrel{(0.13)}{\leq} \mathbb{E}\left[\prod_{|e|=1} \omega(0, e)^{n(e)}\right] \mathbb{E}\left[\prod_{|e|=1} \omega(0, e)^{\widetilde{n}(e)}\right] e^{\alpha \widetilde{n}}
$$

and using symmetry:

$$
\mathbb{E}\left[\prod_{|e|=1} \omega(0, e)^{n(e)+\widetilde{n}(e)}\right] \leq e^{\alpha(n \wedge \widetilde{n})} \mathbb{E}\left[\prod_{|e|=1} \omega(0, e)^{n(e)}\right] \mathbb{E}\left[\prod_{|e|=1} \omega(0, e)^{\tilde{n}(e)}\right]
$$

Inserting this estimate in the last expression of (2.8), we find

$$
\begin{aligned}
\mathbb{E}\left[f_{N}^{2}\right] \leq & \sum_{\sigma, \tilde{\sigma} \in \mathcal{P}_{N}} \prod_{x \in \mathbb{Z}^{d}} e^{\alpha L_{\sigma}(x) \wedge L_{\tilde{\sigma}}(x)} \\
& \mathbb{E}\left[\prod_{|e|=1} \omega(x, e)^{L_{\sigma}(x, e)}\right] \mathbb{E}\left[\prod_{|e|=1} \omega(x, e)^{L_{\tilde{\sigma}}(x, e)}\right] \\
= & \sum_{\sigma, \widetilde{\sigma} \in \mathcal{P}_{N}} e^{\alpha \sum_{x} L_{\sigma}(x) \wedge L_{\tilde{\sigma}}(x)} P_{\sigma(0)}[X \succeq \sigma] P_{\tilde{\sigma}(0)}[X \succeq \tilde{\sigma}] .
\end{aligned}
$$

Note that $L_{\sigma}(x) \wedge L_{\widetilde{\sigma}}(x)=0$, whenever $x \cdot \ell \geq 0$, in view of the last condition in the definition of $\mathcal{P}_{N}$ and (2.7). Proceeding as in (2.6) we find in the notation of (1.13)

$=\sum_{x, \widetilde{x}} E_{x} \otimes E_{\widetilde{x}}\left[A_{N}(0), \widetilde{A}_{N}(0), \exp \left\{\alpha \sum_{z \cdot \ell<0}\left(\sum_{n=0}^{H_{0}-1} 1\left\{X_{n}=z\right\}\right) \wedge\left(\sum_{n=0}^{\widetilde{H}_{0}-1} 1\left\{\widetilde{X}_{n}=z\right\}\right)\right\}\right]$.

Further observe that for an event $E \in \mathcal{F}_{H_{0}}$,

$$
\sum_{x} P_{x}\left[A_{N}(0), E\right]=\sum_{x} P_{x}\left[D^{\ell}>H_{0}, \tau_{N}\left(\bar{X}^{0}\right)=H_{0}, E\right]
$$


but for any $x, P_{x, \omega}\left[D^{\ell}>H_{0}, \tau_{N}\left(\bar{X}^{0}\right)=H_{0}, E\right]$ is $\sigma(\omega(y, \cdot), \ell \cdot y<0)$-measurable, so that multiplying and dividing the above by $P_{0}\left[D^{\ell}=\infty\right]$, using independence, we find:

$$
=\sum_{x} P_{x}\left[X_{\tau_{N}}=0, E \mid D^{\ell}=\infty\right] .
$$

As a result of this calculation and of the renewal property (1.12), we see that

$$
\begin{aligned}
& \text { under the probability } \sum_{x} P_{x}\left[A_{N}(0), \cdot\right],\left(H_{0},\left(X_{\left(H_{0}-\cdot\right)_{+}}\right)\right) \\
& \text {has the same law as }\left(s_{N}, Y_{\cdot \wedge s_{N}}\right) \text { under } Q_{0}(=Q) .
\end{aligned}
$$

Taking the above into account in the last line of (2.10), we find:

$$
\mathbb{E}\left[f_{N}^{2}\right] \leq Q_{0,0}\left[\exp \left\{\alpha \sum_{z} L_{N}(z) \wedge \widetilde{L}_{N}(z)\right\}\right],
$$

which proves (2.4).

REMARK 2.2. 1) The above lemma makes no claim about the finiteness of the expression in the right hand side of (2.4).

2) For certain single site distributions $\mu$, which for instance are finite sums of Dirac masses one can find $\beta>0$, such that for $n(e), \widetilde{n}(e) \geq 0$,

$$
\mathbb{E}\left[\prod_{|e|=1} \omega(0, e)^{n(e)+\tilde{n}(e)}\right] \leq e^{\beta} \mathbb{E}\left[\prod_{|e|=1} \omega(0, e)^{n(e)}\right] \mathbb{E}\left[\prod_{|e|=1} \omega(0, e)^{\tilde{n}(e)}\right] .
$$

This variation of (2.9) then leads to a bound

$$
\mathbb{E}\left[f_{N}^{2}\right] \leq Q_{0,0}\left[\exp \left\{\beta\left|Y_{\left[1, s_{N}\right]} \cap \widetilde{Y}_{\left[1, \widetilde{s}_{N}\right]}\right|\right\}\right], \text { for } N \geq 1,
$$

in place of (2.4), $\left(\left|Y_{\left[1, s_{N}\right]} \cap \widetilde{Y}_{\left[1, \widetilde{s}_{N}\right]}\right|\right.$ denotes the number of sites common to $Y_{n}, 1 \leq$ $n \leq s_{N}$, and $\left.\widetilde{Y}_{n}, 1 \leq n \leq \widetilde{s}_{N}\right)$. This could possibly lead to results in the spirit of the remainder of this section concerning certain examples of nestling walks (for which (0.9) breaks down).

We now introduce some more notations. The function

$$
G(z)=\sum_{i, j \geq 0} Q_{0,0}\left(\widetilde{Y}_{\widetilde{s}_{j}}-Y_{s_{i}}=z\right), z \in \mathbb{Z}^{d},
$$

plays an important role in what follows. We also need

$$
\nu(n)=P_{0}\left[\tau_{2}=n \mid D^{\ell}=\infty\right], n \geq 0,
$$

the law of $\tau_{2}$ under $P_{0}\left[\cdot \mid D^{\ell}=\infty\right]$. We shall now express in terms of $G(\cdot)$ and $\nu(\cdot)$ sufficient conditions which enable us to bound uniformly some exponential moments of $\sum_{x} L_{N}(x) \wedge \widetilde{L}_{N}(x)$. We use the "obvious notation" $L_{\infty}(x)$ in the next proposition.

Proposition 2.3. (under (0.2), (1.5), $1 \leq a \leq 10 \sqrt{d}$ )

If $\rho>0$ and $R>0$ are such that

$$
\begin{aligned}
& \sum_{\widetilde{z}, n} G(\widetilde{z}-z) 1\{|\widetilde{z}| \leq n\} e^{2 \rho n} \nu(n) \leq \frac{1}{2}, \text { for }|z| \geq R, \text { and } \\
& \left(\sum_{n} e^{2 \rho n} \nu(n)\right)^{2 R+1} \leq \frac{3}{2}, \text { then } \\
& Q_{z, \tilde{z}}\left[\exp \left\{\rho \sum_{x} L_{\infty}(x) \wedge \widetilde{L}_{\infty}(x)\right\}\right] \leq 6, \text { for } z, \widetilde{z} \in \mathbb{Z}^{d} .
\end{aligned}
$$


Proof. We pick $N \geq 1$, and first bound $\sum_{x} L_{N}(x) \wedge \widetilde{L}_{N}(x)$. Observe that from the definition of $Q_{z}$, see above (2.3), for any $z \in \mathbb{Z}^{d}$

$$
Q_{z} \text {-a.s., for } n, i \geq 0, Y_{s_{i+1}} \cdot \ell \leq Y_{n} \cdot \ell<Y_{s_{i}} \cdot \ell, \text { when } s_{i}<n \leq s_{i+1} \text {. }
$$

Hence $Q_{z}$-a.s., for any $x \in \mathbb{Z}^{d}$ :

$$
\begin{aligned}
& L_{N}(x)>0 \text { implies that for a unique } i \in[0, N-1], \\
& L_{N}(x)=\sum_{s_{i}<n \leq s_{i+1}} 1\left\{Y_{n}=x\right\} .
\end{aligned}
$$

Therefore, for any $z, \widetilde{z} \in \mathbb{Z}^{d}, Q_{z, \widetilde{z}^{-a . s .:}}$

$$
\begin{aligned}
& \sum_{x} L_{N}(x) \wedge \widetilde{L}_{N}(x)=\sum_{x} \sum_{0 \leq i, j<N}\left(\sum_{s_{i}<n \leq s_{i+1}} 1\left\{Y_{n}=x\right\}\right) \wedge \\
& \left(\sum_{\tilde{s}_{j}<m \leq \widetilde{s}_{j+1}} 1\left\{\widetilde{Y}_{m}=x\right\}\right) .
\end{aligned}
$$

Let us then denote by $I_{i, j}$ the event that the piece of trajectory $Y_{\left(s_{i}, s_{i+1}\right]}$ intersects the piece of trajectory $\widetilde{Y}_{\left(\widetilde{s}_{j}, \widetilde{s}_{j+1}\right]}$ :

$$
I_{i, j}=\left\{\text { for some } n \in\left(s_{i}, s_{i+1}\right], m \in\left(\widetilde{s}_{j}, \widetilde{s}_{j+1}\right], Y_{n}=\widetilde{Y}_{m}\right\} \text {. }
$$

In view of $(2.22)$, we find that $Q_{z, \tilde{z}^{-a . s .}}$

$$
\begin{aligned}
& \sum_{x} L_{N}(x) \wedge \widetilde{L}_{N}(x) \leq \\
& \sum_{0 \leq i, j<N} \sum_{x} 1_{I_{i, j} \cap\left\{Y_{s_{i}} \cdot \ell \geq \widetilde{Y}_{\widetilde{s}_{j}} \cdot \ell\right\}}\left(\sum_{s_{i}<n \leq s_{i+1}} 1\left\{Y_{n}=x\right\}\right) \wedge\left(\sum_{\widetilde{s}_{j}<m \leq \widetilde{s}_{j+1}} 1\left\{\widetilde{Y}_{m}=x\right\}\right)+ \\
& \sum_{0 \leq i, j<N} \sum_{x} 1_{I_{i, j} \cap\left\{\widetilde{Y}_{\widetilde{s}_{j}} \cdot \ell \geq Y_{s_{i}} \cdot \ell\right\}}\left(\sum_{s_{i}<n \leq s_{i+1}} 1\left\{Y_{n}=x\right\}\right) \wedge\left(\sum_{\widetilde{s}_{j}<m \leq \widetilde{s}_{j+1}} 1\left\{\widetilde{Y}_{m}=x\right\}\right) \leq \\
& \sum_{0 \leq i<N}\left(s_{i+1}-s_{i}\right) 1_{\bigcup_{j=0}^{N-1}\left(I_{i, j} \cap\left\{Y_{s_{i}} \cdot \ell \geq \widetilde{Y}_{\widetilde{s}_{j}} \cdot \ell\right\}\right)}+ \\
& \sum_{0 \leq j<N}\left(\widetilde{s}_{j+1}-\widetilde{s}_{j}\right) 1_{\bigcup_{j=0}^{N-1}\left(I_{i, j} \cap\left\{\tilde{Y}_{\widetilde{s}_{j}} \cdot \ell \geq Y_{s_{i}} \cdot \ell\right\}\right)} .
\end{aligned}
$$

Using Cauchy-Schwarz's inequality and symmetry, we find

$$
\begin{aligned}
& \sup _{z, \widetilde{z}} Q_{z, \widetilde{z}}\left[\exp \left\{\rho \sum_{x} L_{N}(x) \wedge \widetilde{L}_{N}(x)\right\}\right] \leq \\
& \sup _{z, \widetilde{z}} Q_{z, \widetilde{z}}\left[\exp \left\{2 \rho \sum_{0 \leq i<N}\left(s_{i+1}-s_{i}\right) 1_{\bigcup_{j=0}^{N-1}\left(I_{i, j} \cap\left\{Y_{s_{i}} \cdot \ell \geq \widetilde{Y}_{\widetilde{s}_{j}} \cdot \ell\right\}\right)}\right\}\right] .
\end{aligned}
$$

We can introduce for $i \geq 0$,

$$
J_{i}=\inf \left\{j \geq 0, \widetilde{Y}_{\widetilde{s}_{j}} \cdot \ell \leq Y_{s_{i}} \cdot \ell\right\},
$$

and using (2.20) we see that the right member of (2.25) equals

$$
\sup _{z, \widetilde{z}} Q_{z, \tilde{z}}\left[\exp \left\{2 \rho \sum_{0 \leq i<N}\left(s_{i+1}-s_{i}\right) 1_{\left\{I_{i j} \text { occurs for some } j \in\left[J_{i}, J_{i+1} \wedge N\right)\right\}}\right] .\right.
$$


Our main claim (2.19) will therefore follow once we show:

$$
\sup _{N \geq 1, M>0, \widetilde{y} \in \mathbb{Z}^{d}} \Psi_{N, M}(\widetilde{y}) \leq 6
$$

with the notation

$$
\Psi_{N, M}(\widetilde{y})=Q_{0, \widetilde{y}}\left[\exp \left\{2 \rho \sum_{0 \leq i<N}\left(\left(s_{i+1}-s_{i}\right) \wedge M\right) 1_{\left\{I_{i j} \text { occurs for some } j \in\left[J_{i}, J_{i+1} \wedge N\right)\right\}}\right\}\right] \text {. }
$$

Let us then define

$$
\begin{aligned}
S= & \inf \left\{i \geq 0, I_{i, j} \text { occurs for some } j \in\left[J_{i}, J_{i+1}\right)\right\} \leq \infty, \\
\widetilde{T}= & \inf \left\{j \in\left[J_{S}, J_{S+1}\right), I_{S, j} \text { occurs }\right\} \leq \infty \\
& (\widetilde{T}=\infty, \text { by convention when } S=\infty)
\end{aligned}
$$

and denote by $\left(\Gamma_{m}\right)_{m \geq 0}$ the shift on the canonical space $\sum$ (where the $Q_{z}$ are defined), which is such that $s_{i} \circ \Gamma_{m}=s_{i+m}-s_{m}, i \geq 0, Y$. $\Gamma_{m}=Y_{s_{m}+}$. Observe that for

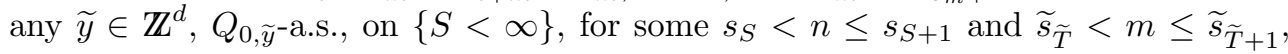
$\tilde{Y}_{\widetilde{s}_{\tilde{T}}} \cdot \ell>\widetilde{Y}_{m} \cdot \ell=Y_{n} \cdot \ell \geq Y_{s_{S+1}} \cdot \ell$.

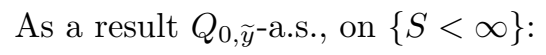

$$
J_{S+1} \geq \widetilde{T}+1, \text { and for } i \geq 0, J_{S+1+i}=J_{i} \circ\left(\Gamma_{S+1} \times \Gamma_{\widetilde{T}+1}\right)+\widetilde{T}+1
$$

Let us then pick $|\widetilde{y}| \geq R$, cf. above (2.17), and write

$$
\begin{aligned}
\Psi_{N, M}(\widetilde{y}) \leq & 1+Q_{0, \widetilde{y}}\left[S<\infty, \exp \left\{2 \rho\left(s_{S+1}-s_{S}\right)\right\} \exp \left\{2 \rho \sum_{0 \leq i<N}\left(s_{i+1}-s_{i}\right)\right.\right. \\
& \left.\left.\wedge M 1_{\left\{I_{i j} \text { occurs for some } j \in\left[J_{i}, J_{i+1} \wedge N\right)\right\}}\right\} \circ\left(\Gamma_{S+1} \times \Gamma_{\widetilde{T}+1}\right)\right] .
\end{aligned}
$$

Observe that for any $\widetilde{y} \in \mathbb{Z}^{d}, i \geq 0, j \geq 0$, the event $\{S=i, \widetilde{T}=j\}$ is $\sigma\left(s_{0}, \ldots, s_{i}, s_{i+1}\right.$, $\left.Y_{s_{i+1} \wedge}\right) \otimes \sigma\left(\widetilde{s}_{0}, \ldots, \widetilde{s}_{j+1}, \widetilde{Y}_{\widetilde{s}_{j+1} \wedge \cdot}\right)$-measurable up to a $Q_{0, \widetilde{y}^{-n e g l i g i b l e ~ s e t . ~ F u r t h e r ~ f o r ~}}$ any $z, Q_{z}$ satisfies the Markov property

$$
Q_{z}\left(A \cap \Gamma_{m}^{-1}(B)\right)=Q_{z}\left(A, Q_{Y_{s_{m}}}(B)\right)
$$

for $m \geq 0$, measurable $B$ and $\sigma\left(s_{0}, \ldots, s_{m}, Y_{s_{m} \wedge}\right.$. $)$-measurable $A$. Applying the Markov property successively with respect to each component, and using translation invariance, we see that the right hand side of (2.32) equals

$$
\begin{aligned}
& 1+Q_{0, \widetilde{y}}\left[S<\infty, \exp \left\{2 \rho\left(s_{S+1}-s_{S}\right)\right\} \Psi_{N, M}\left(\widetilde{Y}_{\widetilde{s}_{\tilde{T}+1}}-Y_{s_{S+1}}\right)\right] \leq \\
& 1+Q_{0, \widetilde{y}}\left[S<\infty, \exp \left\{2 \rho\left(s_{S+1}-s_{S}\right)\right\}\right]\left\|\Psi_{N, M}\right\|_{\infty} .
\end{aligned}
$$

Note that

$$
\begin{aligned}
Q_{0, \widetilde{y}}\left[S<\infty, \exp \left\{2 \rho\left(s_{S+1}-s_{S}\right)\right\}\right] & =\sum_{i \geq 0} Q_{0, \widetilde{y}}\left[S=i, e^{2 \rho\left(s_{i+1}-s_{i}\right)}\right] \\
& \leq \sum_{i \geq 0, j \geq 0} Q_{0, \widetilde{y}}\left[I_{i, j}, e^{2 \rho\left(s_{i+1}-s_{i}\right)}\right]
\end{aligned}
$$




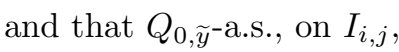

$$
\left|Y_{s_{i}}-\widetilde{Y}_{\widetilde{s}_{j}}\right| \leq s_{i+1}-s_{i}+\widetilde{s}_{j+1}-\widetilde{s}_{j},
$$

since for some $n \in\left(s_{i}, s_{i+1}\right], m \in\left(\widetilde{s}_{j}, \widetilde{s}_{j+1}\right], Y_{n}=\widetilde{Y}_{m}$. As a result we see that

$$
\begin{aligned}
& Q_{0, \tilde{y}}\left[S<\infty, \exp \left\{2 \rho\left(s_{S+1}-s_{S}\right)\right\}\right] \leq \sum_{i, j \geq 0} Q_{0, \tilde{y}}\left[\left|Y_{s_{i}}-\widetilde{Y}_{\widetilde{s}_{j}}\right| \leq\right. \\
& \left.s_{i+1}-s_{i}+\widetilde{s}_{j+1}-\widetilde{s}_{j}, e^{2 \rho\left(s_{i+1}-s_{i}\right)}\right]
\end{aligned}
$$

and from $(2.2),(2.15),(2.16)$, this last quantity is smaller than

$$
\begin{aligned}
\sum_{i, j, n \geq 0} Q_{0, \tilde{y}}\left[\left|Y_{s_{i}}-\widetilde{Y}_{\widetilde{s}_{j}}\right| \leq n, e^{2 \rho n}\right] \nu(n) & =\sum_{z, n} G(z) 1\{|\widetilde{y}+z| \leq n\} e^{2 \rho n} \nu(n) \\
& \leq \frac{1}{2}, \text { in view of }(2.17) \text { and }|\widetilde{y}| \geq R
\end{aligned}
$$

Therefore, when $|\widetilde{y}| \geq R, N \geq 1, M>0$ :

$$
\Psi_{N, M}(\widetilde{y}) \leq 1+\frac{1}{2}\left\|\Psi_{N, M}\right\|_{\infty} .
$$

Pick now $|\widetilde{y}|<R$. Since $a \geq 1, Q$-a.s., $Y_{s_{i}} \cdot \ell \leq-a i \leq-i$. Since $|\widetilde{y}|<R$, defining

$$
i_{0}=[2 R]
$$

we see that

$$
Q \text {-a.s., }\left|Y_{s_{i_{0}+1}}-\widetilde{y}\right| \geq R \text {. }
$$

Further, we can write:

$$
\begin{aligned}
& \Psi_{N, M}(\widetilde{y}) \leq Q_{0, \widetilde{y}}\left[\exp \left\{2 \rho \sum_{i \leq i_{0}}\left(s_{i+1}-s_{i}\right)\right\}\right. \\
& \left.\left.\exp \left\{2 \rho \sum_{0 \leq i<N}\left(s_{i+1}-s_{i}\right) \wedge M 1_{\left\{I_{i j} \text { occurs for some } j \in\left[J_{i}, J_{i+1} \wedge N\right)\right\}}\right]\right\} \circ\left(\Gamma_{i_{0}+1} \times I d\right)\right]
\end{aligned}
$$

and using the Markov property and translation invariance

$$
\begin{aligned}
& =Q_{0, \tilde{y}}\left[\exp \left\{2 \rho \sum_{i \leq i_{0}}\left(s_{i+1}-s_{i}\right)\right\} \Psi_{N, M}\left(\widetilde{y}-Y_{s_{i_{0}+1}}\right)\right] \\
& \stackrel{(2.34)-(2.36),(2.2)}{\leq}\left(1+\frac{1}{2}\left\|\Psi_{N, M}\right\|_{\infty}\right) Q\left[e^{2 \rho s_{1}}\right]^{i_{0}+1} \stackrel{(2.18)}{\leq} \frac{3}{2}+\frac{3}{4}\left\|\Psi_{N, M}\right\|_{\infty} .
\end{aligned}
$$

Combining (2.34) and (2.37), we see that

$$
\left\|\Psi_{N, M}\right\|_{\infty} \leq \frac{3}{2}+\frac{3}{4}\left\|\Psi_{N, M}\right\|_{\infty} .
$$

Further as follows from direct inspection, $\left\|\Psi_{N, M}\right\|_{\infty}<\infty$. The claim (2.27) immediately follows. This completes the proof of Proposition 2.3.

We now come to the main result of this section. From now on we assume (0.9), that is we are in the so-called non-nestling situation, cf. [20], [18], and $\ell \in S^{d-1}$, $\eta>0$, are such that

$$
\mathbb{P} \text {-a.s., for all } x \in \mathbb{Z}^{d}, d(x, \omega) \cdot \ell \geq \eta \text {. }
$$


This ensures in particular that (1.5) holds, cf. Proposition 2.4 of [20] for instance. We keep the restriction on $a$

$$
2<a \leq 10 \sqrt{d}
$$

Theorem 2.4. ( $d \geq 4$, under $(0.2),(0.9),(2.40))$

There exists $\rho_{0}(d, \eta, \kappa)>0$ such that

$$
Q_{z, \widetilde{z}}\left[\exp \left\{\rho_{0} \sum_{x} L_{\infty}(x) \wedge \widetilde{L}_{\infty}(x)\right\}\right] \leq 6, \text { for } z, \widetilde{z} \text { in } \mathbb{Z}^{d}
$$

Further in the notations of (0.13) and Proposition 1.2, when $\alpha \leq \rho_{0}$,

$$
\mathbb{Q} \ll \mathbb{P},
$$

and hence $\mathbb{Q}$ is the only $R$-invariant probability absolutely continuous with respect to $\mathbb{P}$, and it is equivalent to $\mathbb{P}$ and an ergodic $R$-invariant measure.

Proof. Let us explain how the second half of the theorem follows from (2.41). From Lemma 2.1, we see that when $\alpha \leq \rho_{0}, \sup _{N} \mathbb{E}\left[f_{N}^{2}\right]<\infty$. Along some extracted subsequence $N_{k}, f_{N_{k}}$ converges weakly in $L^{2}(\mathbb{P})$ to $f_{\infty}$. It follows from (1.31) that

$$
\mathbb{Q}_{0}=f_{\infty} \frac{P_{0, \omega}\left[D^{\ell}=\infty\right]}{P_{0}\left[D^{\ell}=\infty\right]} \mathbb{P}
$$

The claim (2.42) then follows from (1.29).

The last statement about uniqueness equivalence and ergodicity is classical, see for instance Kozlov [11], p. 82, or Lecture 1 in [3].

We now turn to the proof of (2.41). From Theorem 5.2 in the Appendix,

$$
\sup _{x}\left(1+|x|^{\frac{d-3}{2}}\right) G(x) \leq c_{1}(d, \eta, \kappa)
$$

Further as we now see for some $c_{2}(d, \eta)>0$ :

$$
E_{0}\left[e^{8 c_{2} \tau_{1}} \mid D^{\ell}=\infty\right] \leq \frac{3}{2} .
$$

Indeed as follows from (1.32) of Sznitman [18], for suitable $c_{3}(d, \eta)>0, c_{4}(d, \eta)>1$,

$$
E_{0}\left[\exp \left\{c_{3} X_{\tau_{1}} \cdot \ell\right\}\right] \leq c_{4}
$$

Further for $u>0$,

$$
\begin{aligned}
P_{0}\left[\tau_{1}>u\right] & \leq P_{0}\left[X_{\tau_{1}} \cdot \ell>\frac{\eta}{2} u\right]+P_{0}\left[X_{\tau_{1}} \cdot \ell \leq \frac{\eta}{2}, \tau_{1}>u\right] \\
& \leq c_{4} e^{-c_{3} \frac{\eta}{2} u}+P_{0}\left[T_{\frac{\eta}{2} u}^{\ell}>u\right] .
\end{aligned}
$$

However for $\omega \in \Omega, x \in \mathbb{Z}^{d}$,

$$
M_{n}=X_{n}-X_{0}-\sum_{0}^{n-1} d\left(X_{k}, \omega\right)
$$


is a $P_{x, \omega}$-martingale with bounded increments, and for $\mathbb{P}$-a.e. $\omega$, on $\left\{T_{\frac{\eta}{2} u}^{\ell}>u\right\}$, $P_{0, \omega}$-a.s., $M_{[u]} \cdot \ell \leq \frac{\eta}{2} u-\eta(u-1) \leq-\frac{\eta}{2} u+\eta$. Using Azuma's inequality, cf. AlonSpencer-Erdös [1], p. 85,

$$
P_{0}\left[T_{\frac{\eta}{2} u}^{\ell}>u\right] \leq \exp \left\{-\frac{\eta^{2}}{32 u}(u-2)_{+}^{2}\right\}, \text { for } u>0 .
$$

Moreover from (1.27) of [18], for a suitable $c_{5}(d, \eta)>0$

$$
P_{0}\left[D^{\ell}=\infty\right] \geq c_{5}
$$

Inserting (2.49) in the last member of (2.47), and using (2.50), the claim (2.45) easily follows by choosing $c_{2}$ small enough. From the renewal property (1.12), we find

$$
\sum_{n} e^{4 c_{2} n} \nu(n) \leq \frac{3}{2}
$$

Further for $z \neq 0$, breaking the sum below according to $|\widetilde{z}-z| \geq \frac{1}{2}|z|$ or $|\widetilde{z}-z|<\frac{1}{2}|z|$, we obtain

$$
\begin{aligned}
& \sum_{\widetilde{z}, n} G(\widetilde{z}-z) 1\{|\widetilde{z}| \leq n\} e^{c_{2} n} \nu(n) \stackrel{(2.44)}{\leq} \frac{c_{6}}{|z|^{\frac{d-3}{2}}} \sum_{n}\left(n^{d}+1\right) e^{c_{2} n} \nu(n) \\
& +c_{7} \sum_{n}|z|^{d} 1\left\{\frac{|z|}{2} \leq n\right\} e^{c_{2} n} \nu(n)
\end{aligned}
$$

and using (2.51)

$$
\leq c_{8}\left(|z|^{-\frac{(d-3)}{2}}+e^{-c_{2}|z|}\right)
$$

where all the above positive constants depend at most on $d, \eta, \kappa$. As a result we can choose $R(d, \eta, \kappa)>1$ such that for $|z| \geq R$, the rightmost hand side of (2.52) is smaller than $\frac{1}{2}$. Defining $\rho_{0}$ via

$$
\rho_{0}(2 R+1)=c_{2}, \quad\left(\text { in particular } c_{2} \geq 2 \rho_{0}\right),
$$

we see that $\rho_{0}$ and $R$ fulfill (2.17) - (2.18), and (2.41) follows from (2.19).

REMARK 2.5. Because $\rho_{0}$ solely depends on $d, \eta, \kappa$, it is easy to provide examples where $(0.2)$, (0.9) hold and $\alpha \leq \rho_{0}$. The condition $\alpha \leq \rho_{0}$ can be viewed as an assumption about low disorder for the walk. The above theorem shows the equivalence of the static and dynamic points of views for non-nestling walks when the dimension is high and the disorder is low. The results of Section 3 will naturally lead to question whether these assumptions are mainly artifacts of the proof.

The next result is a variant of Theorem 2.4, which will be used in Section 4 when we prove a "quenched" central limit theorem.

Theorem 2.6. ( $d \geq 4$, under $(0.2),(0.9))$

There exists $\rho_{1}(d, \eta, \kappa)>0$, such that in the notations of $(0.15)$,

$$
E_{x} \otimes E_{\widetilde{x}}\left[\exp \left\{\rho_{1} \sum_{z} L_{X}(z) \wedge L_{\widetilde{X}}(z)\right\}\right] \leq 10, \text { for } x, \widetilde{x} \in \mathbb{Z}^{d} .
$$

Proof. Let us define for $x, \widetilde{x} \in \mathbb{Z}^{d}$

$$
\varphi(x, \widetilde{x})=E_{x} \otimes E_{\widetilde{x}}\left[\exp \left\{\rho_{0} \sum_{z} L_{X}(z) \wedge L_{\widetilde{X}}(z)\right\} \mid D^{\ell}=\infty, \widetilde{D}^{\ell}=\infty\right] .
$$


Choosing $a$ as in (2.40), the renewal property (1.12) and a mere repetition of the arguments of Proposition 2.3 and of the proof of (2.41) shows that

$$
\|\varphi\|_{\infty} \leq 6
$$

(the sole modification is that we replace $I_{i, j}$ in $(2.23)$ by

$$
\mathcal{I}_{i, j}=\left\{\text { for some } n \in\left[\tau_{i}, \tau_{i+1}\right), m \in\left[\widetilde{\tau}_{j}, \widetilde{\tau}_{j+1}\right), X_{n}=\widetilde{X}_{m}\right\}
$$

and distinguish between cases where $X_{\tau_{i}} \cdot \ell \geq \widetilde{X}_{\widetilde{\tau}_{j}} \cdot \ell$ or $\widetilde{X}_{\widetilde{\tau}_{j}} \cdot \ell \geq X_{\tau_{i}} \cdot \ell$ in the analogue of (2.24)). Consider $\rho_{1} \leq \rho_{0}$, we see that the expression in (2.54) is smaller than:

$$
E_{x} \otimes E_{\widetilde{x}}\left[\exp \left\{\rho_{1}\left(\tau_{1}+\widetilde{\tau}_{1}\right)\right\} \exp \left\{\rho_{0} \sum_{z} L_{X}(z) \wedge L_{\widetilde{X}}(z)\right\} \circ\left(\theta_{\tau_{1}} \times \theta_{\widetilde{\tau}_{1}}\right)\right]
$$

and applying the renewal property (1.13)

$$
=E_{x} \otimes E_{\widetilde{x}}\left[\exp \left\{\rho_{1}\left(\tau_{1}+\widetilde{\tau}_{1}\right) \varphi\left(X_{\tau_{1}}, \widetilde{X}_{\widetilde{\tau}_{1}}\right)\right] \leq 6 E_{0}\left[e^{\rho_{1} \tau_{1}}\right]^{2}\right.
$$

and from $(2.47),(2.49)$, we see that we can choose $\rho_{1}(d, \eta, \kappa)$ so that this last term is smaller than 10 .

REMARK 2.7. The above applies in particular to the case of a classical nearest neighbor simple random walk with jump probability $(p(e))_{|e|=1}$, when $d \geq 4, p(e)>0$ for each $e$, and $\sum_{|e|=1}$ e $p(e) \neq 0$. It shows that for $\rho=\rho_{1}\left(d, \eta=\left|\sum e p(e)\right|, \kappa=\right.$ $\left.\inf _{e} p(e)\right)$, for all $x, \widetilde{x} \in \mathbb{Z}^{d}$,

$$
E_{x} \otimes E_{\widetilde{x}}\left[\exp \left\{\rho \sum_{z} L_{X}(z) \wedge L_{\widetilde{X}}(z)\right\}\right] \leq 10 .
$$

3. The directed case revisited. In this section, we return to the case of directed walks and show that the counterexample discussed at the end of Section I is unstable. From now on, we assume (0.3) as well as

$$
\mathbb{P}\left[\omega\left(0, e_{i}\right)>0\right]=1, \text { for } 1 \leq i \leq d .
$$

For directed walks the one-dimensional situation is of course trivial, and we only focus on the case $d \geq 2$. With the notations of (1.34) and Proposition 1.4, our main object is

Theorem 3.1. ( $d \geq 2$, under $(0.3),(3.1))$

$$
\sup _{\mathbb{N}} \mathbb{E}\left[g_{N}^{2}\right]<\infty
$$

$$
\mathbb{Q} \ll \mathbb{P}
$$

Proof. The claim (3.3) follows from (3.2) and Proposition 1.4. Indeed $g_{N}$ is a square integrable martingale which converges in $L^{2}(\mathbb{P})$ and $\mathbb{P}$-a.s. to $g_{\infty}$, and $\mathbb{Q}=g_{\infty} \mathbb{P}$. We turn to the proof of (3.2). For $N \geq 1$, 


$$
\begin{aligned}
\mathbb{E}\left[g_{N}^{2}\right]= & \sum_{x, \widetilde{x}} \mathbb{E}\left[p_{N}(x, 0, \omega) p_{N}(\widetilde{x}, 0, \omega)\right]= \\
& \sum_{x, \widetilde{x}} \mathbb{E}\left[P_{x, \omega} \otimes P_{\widetilde{x}, \omega}\left[X_{N}=0=\widetilde{X}_{N}\right]\right] \stackrel{\begin{array}{c}
\text { translation } \\
\text { invariance }
\end{array}}{=} \\
& \sum_{x, \widetilde{x}} \mathbb{E}\left[P_{x-\widetilde{x}, \omega} \otimes P_{0, \omega}\left[X_{N}=\widetilde{X}_{N}=-\widetilde{x}\right]\right]=\sum_{y} \mathbb{E}\left[P_{y, \omega} \otimes P_{0, \omega}\left[Z_{N}=0\right]\right],
\end{aligned}
$$

provided $\left(Z_{n}\right)_{n \geq 0}=\left(X_{n}-\widetilde{X}_{n}\right)_{n \geq 0}$. Observe that the summands in the last sum vanish when $y$ does not belong to

$$
\mathcal{L}=\left\{x \in \mathbb{Z}^{d}, x_{1}+\cdots+x_{d}=0\right\} .
$$

Notice also that for $z \in \mathcal{L}$, under $\mathbb{P} \times\left(P_{z, \omega} \otimes P_{0, \omega}\right),\left(Z_{n}\right)_{n \geq 0}$ defines a Markov chain with state space $\mathcal{L}$, starting at point $z$, and with transition kernel

$$
\begin{aligned}
K(x, y)= & \sum_{1}^{d} \mathbb{E}\left[\omega\left(0, e_{i}\right)\right]^{2}, \text { if } x \neq 0 \text { and } y=x \\
& \mathbb{E}\left[\omega\left(0, e_{i}\right)\right] \mathbb{E}\left[\omega\left(0, e_{j}\right)\right], \text { if } x \neq 0 \text { and } y=x+e_{i}-e_{j}, \text { for } i \neq j, \\
& \sum_{1}^{d} \mathbb{E}\left[\omega\left(0, e_{i}\right)^{2}\right], \text { if } x=0 \text { and } y=x, \\
& \mathbb{E}\left[\omega\left(0, e_{i}\right) \omega\left(0, e_{j}\right)\right], \text { if } x=0 \text { and } y=e_{i}-e_{j}, \text { for } i \neq j,
\end{aligned}
$$

0 in all other cases.

Observe that $K$ differs at the single site 0 from the kernel $K_{0}$ of a random walk on $\mathcal{L}$ defined by the first two lines of (3.6), with $x$ arbitrary in $\mathcal{L}$ instead of $x \neq 0$. In view of (3.1) these two kernels are irreducible, also they are either both transient or both recurrent. Note also that by (3.4),

$$
\mathbb{E}\left[g_{N}^{2}\right]=\sum_{x \in \mathcal{L}} K^{N}(x, 0) .
$$

Let us first assume that $K$ is transient. If $\lambda$ stands for the counting measure on $\mathcal{L}$ and $W$ for the Green kernel:

$$
W(x, y)=\sum_{n \geq 0} K^{n}(x, y), x, y \in \mathcal{L},
$$

we define

$$
\Delta(y)=\lambda K(y)-1, y \in \mathcal{L} .
$$

In view of (3.6),

$$
\Delta(y)=0 \text {, whenever } y \notin\left\{e_{i}-e_{j}, 1 \leq i, j \leq d\right\} .
$$

Observe that:

$$
(\lambda+\Delta W) K=\lambda K+\Delta(W-I) \stackrel{(3.9)}{=} \lambda+\Delta+\Delta W-\Delta=\lambda+\Delta W .
$$


Hence iterating the above equality:

$$
(\lambda+\Delta W) K^{N}=\lambda+\Delta W, \text { for } N \geq 0 .
$$

As mentioned above the random walk with kernel $K_{0}$ is transient as well, and necessarily $d \geq 3$. If $W_{0}$ denotes the Green kernel attached to $K_{0}$, we know from Spitzer [17], p. 281 that $\lim _{x \rightarrow \infty} W_{0}(0, x)=0$. From the perturbation formula $W=W_{0}+W\left(K-K_{0}\right) W_{0}$, we infer that

$$
\lim _{x \rightarrow \infty} W(0, x)=0 .
$$

From the identity $W=W_{0}+W_{0}\left(K-K_{0}\right) W$, we see that (3.13) holds with 0 replaced by any point. Hence for some $M>0$,

$$
(\lambda+\Delta W)(y) \geq \frac{1}{2}, \text { when }|y| \geq M .
$$

Coming back to (3.12), we see that for $N \geq 1$,

$$
\begin{aligned}
\frac{1}{2} \sum_{|x| \geq M} K^{N}(x, 0) \leq \sum_{|x| \geq M}(\lambda+\Delta W) K^{N}(0) & =(\lambda+\Delta W)(0)-\sum_{|x|<M}(\lambda+\Delta W) K^{N}(x, 0) \\
& \leq \text { const. }<\infty .
\end{aligned}
$$

From (3.7), we now deduce (3.2).

Let us then assume that $K$ is recurrent. The corresponding invariant measures $\rho$ of $K$ and $\lambda$ of $K_{0}$ are unique up to a constant multiplicative factor, cf. Durrett [6], p. 263. Using the "cycle trick" based at the point 0 , cf. [6], p. 262, in order to express $\frac{\rho(\cdot)}{\rho(0)}$ and $\frac{\lambda(\cdot)}{\lambda(0)} \equiv 1$, we see that

$$
\rho(y) \geq \text { const. }>0, \text { for } y \in \mathcal{L} .
$$

Since for all $N \geq 1$

$$
\sum_{y \in \mathcal{L}} \rho(y) K^{N}(y, 0)=\rho(0),
$$

the claim (3.7) follows. This proves (3.2).

REMARK 3.2. 1) The directed situation is reminiscent of directed polymers in a random potential, cf. Bolthausen [2], Carmona-Molchanov [4], Sinai [16]. However in the case of directed random walks in random environment, what might be nicknamed the "disordered phase", i.e. when $\mathbb{Q}$ is not absolutely continuous with respect to $\mathbb{P}$, see Proposition 1.5, appears to be unstable. Remarkably, independently of the dimension, under the mild partial ellipticity condition (3.1), the second moments $\mathbb{E}\left[g_{N}^{2}\right]$ are uniformly bounded. One can naturally wonder whether this also happens in the fully elliptic situation discussed in Section 2.

2) A straightforward modification of the argument used to prove (1.8), (1.9) in Lecture 1 of [3], shows that under the assumptions of Theorem 3.1, $\mathbb{Q}$ is in fact equivalent to $\mathbb{P}$. It is the only $R$-invariant probability absolutely continuous with respect to $\mathbb{P}$, and the Markov chain with initial law $\mathbb{Q}$ and transition kernel $R$ is ergodic. 
4. A P-almost sure central limit theorem. In this section we apply the controls derived in Section 2, to the derivation of a functional central limit theorem for the $D\left(\mathbb{R}_{+}, \mathbb{R}^{d}\right)$-valued variable

$$
B_{\cdot}^{n}=\frac{1}{\sqrt{n}}\left(X_{[\cdot n]}-[\cdot n] v\right), \text { with } v \text { as in }(0.16),
$$

under $P_{0, \omega}$, for $\mathbb{P}$-a.e. $\omega$. Under quite general assumptions, cf. [18], [19], and in particular under $(0.2),(0.9)$, it is known that

the law of $B^{n}$. under $P_{0}$ converges weakly to the law $P$ of a $d$-dimensional

Brownian motion with non-degenerate covariance matrix $A$.

We shall see that under $(0.2),(0.9)$, when $d \geq 4$, in the low noise regime this central limit theorem under $P_{0}$ can be extended to a $\mathbb{P}$-almost sure central limit theorem under $P_{0, \omega}$. Our main tool to this end is the control of the $\mathbb{P}$-variances of certain $P_{0, \omega}$-expectations. We shall combine these "concentration estimates" with (4.2). It will be convenient to consider the $C\left(\mathbb{R}_{+}, \mathbb{R}^{d}\right)$-valued variable:

$$
\beta_{\cdot}^{n}=\text { the polygonal interpolation of } \frac{k}{n} \longrightarrow B_{\frac{k}{n}}^{n}, k \geq 0 .
$$

Throughout this section the space $C\left(\mathbb{R}_{+}, \mathbb{R}^{d}\right)$ of continuous $\mathbb{R}^{d}$-valued functions on $\mathbb{R}_{+}$is endowed with the topology of uniform convergence on bounded intervals and its Borel $\sigma$-algebra. The space $D\left(\mathbb{R}_{+}, \mathbb{R}^{d}\right)$ is endowed with the Skorohod topology and its Borel $\sigma$-algebra. It is also convenient to endow the space $C\left([0, T], \mathbb{R}^{d}\right)$, for $T>0$, with the distance

$$
d_{T}\left(w, w^{\prime}\right)=\sup _{s \leq T}\left|w(s)-w^{\prime}(s)\right| \wedge 1
$$

and its corresponding Borel $\sigma$-algebra. The next lemma highlights the interest of controlling the $\mathbb{P}$-variance of functionals of the type $E_{0, \omega}\left[F\left(\beta^{n}\right)\right]$, for $F$ an arbitrary bounded Lipschitz function on $C\left([0, T], \mathbb{R}^{d}\right)$, (with a slight abuse of notations concerning $\left.F\left(\beta_{.}^{n}\right)\right)$.

LEMMA 4.1. Assume that for all $T>0$, for all bounded Lipschitz functions $F$ on $C\left([0, T], \mathbb{R}^{d}\right)$, and all $b \in(1,2]$ :

$$
\sum_{m} \operatorname{var}_{\mathbb{P}}\left(E_{0, \omega}\left[F\left(\beta^{\left[b^{m}\right]}\right)\right]\right)<\infty,
$$

then $\mathbb{P}$-a.s.,

$$
\begin{aligned}
& \beta_{.}^{n} \text { converges in law under } P_{0, \omega} \text { towards } P \\
& B_{.}^{n} \text { converges in law under } P_{0, \omega} \text { towards } P .
\end{aligned}
$$

Proof. The statement (4.7) follows from (4.6) and Proposition 10.4, p. 149 in Ethier-Kurtz [8]. Let us prove (4.6). We use the notation $b_{m}$ in place of $\left[b^{m}\right]$. From (4.2), (4.5), and Proposition 10.4 of [8], on a set of full $\mathbb{P}$-measure $\Omega_{0}$, for all rationals $b \in(1,2]$, and functions $F$ of the form

$$
F_{T, \delta}(w)=\sup _{0 \leq s, t \leq T,|t-s| \leq \delta}|w(s)-w(t)| \wedge 1, T>0, \delta>0, \text { rationals, }
$$


or $F$ finite product of functions $\exp \{i z \cdot w(s)\}, z \in \mathbb{Q}^{d}, s>0$ rational,

$$
E_{0, \omega}\left[F\left(\beta^{b_{m}}\right)\right] \rightarrow E^{P}[F], \text { as } m \rightarrow \infty .
$$

Observe that for $T>0$,

$$
h_{T}(\delta) \stackrel{\text { Def }}{=} E^{P}\left[F_{T, \delta}\right] \text {, is such that } \lim _{\delta \rightarrow 0} h_{T}(\delta)=0 \text {. }
$$

This and (4.9) shows that for $\omega \in \Omega_{0}$, for all rationals $b \in(1,2], T \in \mathbb{N}$ :

$$
\text { for all } \epsilon>0 \text {, there exist } \delta>0, \varlimsup_{m} P_{0, \omega}\left[\sup _{\substack{0 \leq s, t \leq T \\|s-t| \leq \delta}}\left|\beta_{s}^{b_{m}}-\beta_{t}^{b_{m}}\right| \geq \epsilon\right] \leq \epsilon .
$$

Hence for $\omega \in \Omega_{0}$, and $b \in(1,2]$, the laws of $\beta^{b_{m}}$ under $P_{0, \omega}$ are tight. An analogous argument with $F$ of the form indicated on the line below (4.8) shows that for $\omega \in \Omega_{0}$,

(4.12) for rational $b \in(1,2]$, the law of $\beta^{b_{m}}$ under $P_{0, \omega}$ converges weakly to $P$.

We now want to infer the convergence of the laws of the $\beta_{\text {. }}^{n}$ under $P_{0, \omega}$. We consider $b$ rational in $(1,2]$, and

$$
b_{m} \leq n<b_{m+1} .
$$

We have the identity:

$$
\beta_{s}^{n}=\sqrt{\frac{b_{m}}{n}} \beta_{\frac{n}{b_{m}}}^{b_{m}}, s \geq 0 .
$$

Hence from (4.9), with $b=2$ and $F=F_{T, \delta}$, we see that for $\omega \in \Omega_{0}, T, \delta>0$ rationals:

$$
\begin{aligned}
& \varlimsup_{n} E_{0, \omega}\left[\sup _{\substack{0 \leq s, t \leq T \\
|s-t| \leq \delta}}\left|\beta_{s}^{n}-\beta_{t}^{n}\right| \wedge 1\right] \leq \varlimsup_{n} E_{0, \omega}\left[\sup _{\substack{0 \leq s, t \leq T \\
|s-t| \leq \delta}}\left|\beta_{s \frac{n}{b_{m}}}^{b_{m}}-\beta_{t \frac{n}{b_{m}}}^{b_{m}}\right| \wedge 1\right] \leq \\
& \varlimsup_{n} E_{0, \omega}\left[\sup _{\substack{0 \leq u, v \leq 2 T \\
|u-v| \leq 2 \delta}}\left|\beta_{u}^{b_{m}}-\beta_{v}^{b_{m}}\right| \wedge 1\right] \stackrel{(4.9)-(4.10)}{=} h_{2 T}(2 \delta) .
\end{aligned}
$$

By a similar argument as in (4.11), we see that for $\omega \in \Omega_{0}$, the laws of the $\beta^{n}$. under $P_{0, \omega}$ are tight. Further with $F$ as in the line below (4.8), $\omega \in \Omega_{0}$, and a large enough rational $T$,

$$
\begin{aligned}
& \varlimsup_{n}\left|E_{0, \omega}\left[F\left(\beta^{n}\right)\right]-E_{0, \omega}\left[F\left(\beta_{\cdot}^{b_{m}}\right)\right]\right| \leq\|F\|_{\operatorname{Lip}} \varlimsup_{n} E_{0, \omega}\left[\sup _{s \leq T}\left|\beta_{s}^{n}-\beta_{s}^{b_{m}}\right| \wedge 1\right] \\
& \stackrel{(4.14)}{=} \varlimsup_{n} E_{0, \omega}\left[\sup _{s \leq T}\left|\sqrt{\frac{b_{m}}{n}} \beta_{s \frac{n}{b_{m}}}^{b_{m}}-\beta_{s}^{b_{m}}\right| \wedge 1\right] \stackrel{(4.10)-(4.12)}{\leq} h_{2 T}((b-1) T)+ \\
& E^{P}\left[\sup _{0 \leq s \leq 2 T}\left|(\sqrt{b}-1) Z_{s}\right| \wedge 1\right],
\end{aligned}
$$

provided $\left(Z_{s}\right)_{s \geq 0}$ denotes the canonical process on $C\left(\mathbb{R}_{+}, \mathbb{R}^{d}\right)$. Letting $b$ tend to 1 , we see that for $\omega \in \Omega_{0}$, (4.6) holds. This concludes the proof of Lemma 4.1. 
We are now ready for the main result of this section. We recall that $\alpha$ is defined in $(0.13)$ and $\rho_{1}$ in $(2.54)$.

TheOREM 4.2. ( $d \geq 4$, under $(0.2),(0.9))$

There exists $\rho_{2}(d, \eta, \kappa)>0$, such that when $\alpha \leq \rho_{2}, \mathbb{P}$-a.s.,

$$
B^{n} \text { converges in law under } P_{0, \omega} \text { to } P \text {, (cf. (4.2) for the definition of } P \text { ). }
$$

Proof. In view of Lemma 4.1, it suffices to show that (4.5) holds whenever $b \in$ $(1,2]$, and $F$ is a function bounded by 1 , with Lipschitz constant smaller than 1 , relative to $d_{T}$. The heart of the matter is to estimate

$$
V^{n}=\operatorname{var}_{\mathbb{P}}\left(E_{0, \omega}\left[F\left(\beta_{.}^{n}\right)\right]\right), n \geq 1 .
$$

We use the martingale method. We pick $a \in(2,10 \sqrt{d}]$, and introduce the sequence $\tau_{k}, k \geq 1$, corresponding to $\ell$ from $(0.9)$ and $a$, as in (1.10), (1.11). We define for $n \geq 1$, the discrete ball

$$
C_{n}=\left\{x \in \mathbb{Z}^{d},|x| \leq n^{\gamma}\right\} \text {, where } \gamma \in\left(0, \frac{1}{2}\right) \text { is some fixed constant }
$$

We introduce an $n$-dependent enumeration of $\mathbb{Z}^{d}$, for which:

$$
z_{m} \in C_{n} \text {, for } m \leq\left|C_{n}\right| \text {, (and hence } z_{m} \notin C_{n} \text { for } m>\left|C_{n}\right| \text { ), }
$$

as well as the $n$-dependent filtration $\left(\mathcal{G}_{k}\right)_{k \geq 0}$ :

$$
\mathcal{G}_{k}=\sigma\left(\omega\left(z_{m}, \cdot\right), m \leq k\right), k \geq 1, \text { and } \mathcal{G}_{0}=\{\emptyset, \Omega\}
$$

We now have the martingale

$$
M_{k}^{n}=\mathbb{E}\left[E_{0, \omega}\left[F\left(\beta^{n}\right)\right] \mid \mathcal{G}_{k}\right], k \geq 0,
$$

so that denoting by $\Delta M_{k}^{n}, k \geq 1$, the increments $M_{k}^{n}-M_{k-1}^{n}$,

$$
V^{n}=\sum_{k \geq 1} \mathbb{E}\left[\left(\Delta M_{k}^{n}\right)^{2}\right]=V_{1}^{n}+V_{2}^{n}
$$

where $V_{1}^{n}, V_{2}^{n}$ respectively refer to the sum restricted to $k \leq\left|C_{n}\right|$ and $k>\left|C_{n}\right|$. From now on we drop the superscript $n$ for simplicity, and start to bound $V_{1}$. We define

$$
\begin{aligned}
m_{n}= & {\left[\frac{n^{\gamma}}{a}\right]+1 } \\
\widetilde{\beta}^{n}= & \beta_{\cdot+}^{n} \frac{\tau_{m_{n}}}{n}-\beta_{\frac{\tau m_{n}}{n}}^{n}=\text { the polygonal interpolation of } \\
& \frac{k}{n} \rightarrow \frac{1}{\sqrt{n}}\left(X_{k+\tau_{m_{n}}}-X_{\tau_{m_{n}}}-k v\right),
\end{aligned}
$$

as well as the variables $\widetilde{M}_{k}$, just as in $(4.21)$, with $\widetilde{\beta}^{n}$ in place of $\beta^{n}$. Note that $P_{0}$-a.s.,

$$
\sup _{s \geq 0}\left|\widetilde{\beta}_{s}^{n}-\beta_{s}^{n}\right| \leq \frac{2}{\sqrt{n}}\left(\tau_{m_{n}}+1\right)
$$


Further we can write with hopefully obvious notations:

$$
\begin{aligned}
V_{1} & \leq \quad 2 \sum_{1 \leq k \leq\left|C_{n}\right|}\left(\mathbb{E}\left[\left(\Delta M_{k}-\Delta \widetilde{M}_{k}\right)^{2}\right]+\mathbb{E}\left[\left(\Delta \widetilde{M}_{k}\right)^{2}\right]\right) \\
& \leq 2 \mathbb{E}\left[\left(M_{\left|C_{n}\right|}-\widetilde{M}_{\left|C_{n}\right|}\right)^{2}\right]+2 \sum_{k \leq\left|C_{n}\right|} \mathbb{E}\left[\left(\Delta \widetilde{M}_{k}\right)^{2}\right] \\
& \leq 2 E_{0}\left[\left(F\left(\beta_{.}^{n}\right)-F\left(\widetilde{\beta}_{\cdot}^{n}\right)\right)^{2}\right]+2 \sum_{k \leq\left|C_{n}\right|} \mathbb{E}\left[\left(\Delta \widetilde{M}_{k}\right)^{2}\right] \\
& \leq \frac{4}{n} E_{0}\left[\left(\tau_{m_{n}}+1\right)^{2}\right]+2 \sum_{k \leq\left|C_{n}\right|} \mathbb{E}\left[\left(\Delta \widetilde{M}_{k}\right)^{2}\right]
\end{aligned}
$$

where we used (4.25) and the Lipschitz property of $F$ in the last step. Further in view of the renewal property (1.12) and Theorem 2.1 of [18] we see that for some constant $c$ independent of $n$

$$
\leq \frac{c}{n} m_{n}^{2}+2 \sum_{1 \leq k \leq\left|C_{n}\right|} \mathbb{E}\left[\left(\Delta \widetilde{M}_{n}\right)^{2}\right]
$$

The conditional expectation with respect to $\mathcal{G}_{k}$ is obtained by integrating out the i.i.d. variable $\omega\left(z_{m}, \cdot\right), m>k$. Denoting by $\mathbb{E}^{\mathcal{G}_{k}}$, this conditional expectation, we find for $0 \leq k \leq\left|C_{n}\right|$ :

$$
\widetilde{M}_{k}=\mathbb{E}^{\mathcal{G}_{k}}\left[E_{0, \omega}[F(\widetilde{\beta} .)]\right]=\sum_{x} \mathbb{E}^{\mathcal{G}_{k}}\left[E_{0, \omega}\left[F(\widetilde{\beta} .), X_{\tau_{m_{n}}}=x\right]\right]
$$

and using the notations of (1.13),

$$
=\sum_{x} \mathbb{E}^{\mathcal{G}_{k}}\left[E_{0, \omega}\left[F(\widetilde{\beta} .), \tau_{m_{n}}\left(\bar{X}_{.}^{x}\right)=H_{x}, D^{\ell} \circ \theta_{H_{x}}=\infty\right]\right]
$$

and in view of (4.24) and the Markov property:

$$
=\sum_{x} \mathbb{E}^{\mathcal{G}_{k}}\left[P_{0, \omega}\left[\tau_{m_{n}}\left(\bar{X}_{0}^{x}\right)=H_{x}\right] E_{x, \omega}\left[D^{\ell}=\infty, F\left(\beta^{n}-\frac{x}{\sqrt{n}}\right)\right]\right] .
$$

Note that in the above sum only sites $x$ contribute for which

$$
x \cdot \ell \geq m_{n} a \stackrel{(4.23)}{>} n^{\gamma}
$$

which are necessarily of the form $z_{m}$ with $m>\left|C_{n}\right|$. Further the term $P_{0, \omega}\left[\tau_{m_{n}}\left(\bar{X}^{x}\right)=\right.$ $\left.H_{x}\right]$ is $\sigma(\omega(z, \cdot) ; z \cdot \ell<x \cdot \ell)$-measurable, whereas $E_{x, \omega}\left[D^{\ell}=\infty, F\left(\beta^{n}-\frac{x}{\sqrt{n}}\right)\right]$ is $\sigma(\omega(z, \cdot), z \cdot \ell \geq x \cdot \ell)$-measurable, and in view of the above discussion, all such $z$ with $z \cdot \ell \geq x \cdot \ell$, are of the form $z_{m}$, with $m>\left|C_{n}\right|$. Hence performing the integration with respect to all variable $\omega(z, \cdot), z \cdot \ell \geq x \cdot \ell$, in the last line of $(4.27)$, we find:

$$
=\sum_{x} \mathbb{E}^{\mathcal{G}_{k}}\left[P_{0, \omega}\left[\tau_{m_{n}}\left(\bar{X}_{.}^{x}\right)=H_{x}\right]\right] E_{0}\left[D^{\ell}=\infty, F\left(\beta_{.}^{n}\right)\right]
$$

but the same calculation with $F=1$, shows that

$$
=E_{0}\left[F\left(\beta_{.}^{n}\right) \mid D^{\ell}=\infty\right]
$$


In particular the rightmost term of (4.26) vanishes and

$$
V_{1} \leq \frac{c}{n} m_{n}^{2}, \text { with } c \text { independent of } n .
$$

We now bound $V_{2}$. We first consider $k>\left|C_{n}\right|, \omega, \omega^{\prime} \in \Omega$ which coincide except perhaps at $z_{k}$, and for which the drift condition (0.9) holds at each site of $\mathbb{Z}^{d}$.

$$
\begin{aligned}
& \left|E_{0, \omega^{\prime}}\left[F\left(\beta^{n}\right)\right]-E_{0, \omega}\left[F\left(\beta^{n}\right)\right]\right|= \\
& \left|E_{0, \omega}\left[F\left(\beta^{n}\right)\left(\prod_{|e|=1}\left(\frac{\omega^{\prime}\left(z_{k}, e\right)}{\omega\left(z_{k}, e\right)}\right) \sum_{0}^{[T n]+1} 1\left\{X_{m}=z_{k}, X_{m+1}=z_{k}+e\right\}-1\right)\right]\right| \text {, }
\end{aligned}
$$

where we recall that $F$ is a function on $C\left([0, T], \mathbb{R}^{d}\right)$ bounded by 1 , so that in the notations of Theorem 2.6, and (0.13),

$$
\leq P_{0, \omega}\left[H\left(z_{k}\right)<\infty, e^{\alpha L_{X}\left(z_{k}\right)}\right]=P_{0, \omega}\left[H\left(z_{k}\right)<\infty\right] E_{z_{k}, \omega}\left[e^{\alpha L_{X}\left(z_{k}\right)}\right] .
$$

In view of $(0.9)$, it is routine to prove that for some $\rho_{3}(d, \eta, \kappa)>0, \rho_{4}(d, \eta, \kappa)>1$

$$
\mathbb{P} \text {-a.s., for all } z \in \mathbb{Z}^{d}, E_{z, \omega}\left[e^{\rho_{3} L_{X}\left(z_{k}\right)}\right] \leq \rho_{4} \text {. }
$$

Therefore combining (4.29), (4.30), we see that provided $\alpha \leq \rho_{3}$

$$
V_{2} \leq c \sum_{k>\left|C_{n}\right|} \mathbb{E}\left[P_{0, \omega}\left[H\left(z_{k}\right)<\infty\right]^{2}\right]
$$

where $c$ has the same meaning as in (4.28). By a similar calculation as in (2.8) $(2.12)$,

$$
\begin{aligned}
& \leq c \sum_{k>\left|C_{n}\right|} E_{0} \otimes E_{0}\left[\exp \left\{\alpha \sum_{x} L_{X}(x) \wedge L_{\widetilde{X}}(x)\right\}, H\left(z_{k}\right)<\infty, \widetilde{H}\left(z_{k}\right)<\infty\right] \\
& \leq c E_{0} \otimes E_{0}\left[\exp \left\{\alpha \sum_{x} L_{X}(x) \wedge L_{\tilde{X}}(x)\right\}\left(\sum_{x} L_{X}(x) \wedge \widetilde{L}_{\tilde{X}}(x)\right), I^{n}\right],
\end{aligned}
$$

where $I^{n}$ stands for the event, in the notation of (1.2),

$$
I^{n}=\left\{X_{i}=\widetilde{X}_{j}, \text { for some } i \geq T_{C_{n}}, j \geq \widetilde{T}_{C_{n}}\right\} .
$$

If we apply Cauchy-Schwarz's inequality to the last expression of (4.31), and use Theorem 2.6, the bound we obtain combined with (4.28), shows that when $\alpha<\frac{\rho_{1}}{2} \wedge \rho_{3}$, for any $b \in(1,2]$ and function $F$ on $C\left([0, T], \mathbb{R}^{d}\right)$ bounded by 1 , with Lipschitz constant 1 with respect to $d_{T},(4.5)$ holds, provided we can show that for some $\zeta>0$,

$$
\varlimsup_{n} n^{\zeta} P_{0} \otimes P_{0}\left[I^{n}\right]<\infty .
$$

By Proposition 1.4 of [18], $\sup _{0 \leq m \leq \tau_{1}}\left|X_{m}\right|$ has some finite exponential moment under $P_{0}$, so that by standard Cramer-type estimates, and the renewal property (1.12), for small $c>0$ :

$$
\varlimsup_{n} n^{-\gamma} \log P_{0}\left[T_{C_{n}}<\tau_{m_{n}^{\prime}}\right]<0, \text { with } m_{n}^{\prime}=\left[c n^{\gamma}\right] .
$$

The claim (4.33) will thus follow if we show that for some $\zeta>0$,

$$
\begin{aligned}
& \varlimsup n^{\zeta} P_{0} \otimes P_{0}\left[J^{n}\right]<\infty, \text { with } \\
& J^{n}=\left\{X_{i}=\widetilde{X}_{j}, \text { for some } i \geq \tau_{m_{n}^{\prime}}, j \geq \widetilde{\tau}_{m_{n}^{\prime}}\right\} .
\end{aligned}
$$


Clearly for $R>0$,

$$
\begin{aligned}
& P_{0} \otimes P_{0}\left[J^{n}\right] \leq P_{0} \otimes P_{0}\left[\left|X_{\tau_{m_{n}^{\prime}}}-\widetilde{X}_{\widetilde{\tau}_{m_{n}^{\prime}}}\right| \leq R\right]+ \\
& \sum_{i, j \geq 0} P_{0} \otimes P_{0}\left[\left|X_{\tau_{m_{n}^{\prime}}}-\widetilde{X}_{\widetilde{\tau}_{m_{n}^{\prime}}}\right|>R, X_{\left[\tau_{m_{n}^{\prime}+i}, \tau_{m_{n}^{\prime}+i+1}\right)} \cap \widetilde{X}_{\left[\widetilde{\tau}_{m_{n}^{\prime}+j}, \widetilde{\tau}_{m_{n}^{\prime}+j+1}\right)} \neq \emptyset\right],
\end{aligned}
$$

so by a similar argument as in (2.33),

$$
\leq P_{0} \otimes P_{0}\left[\left|X_{\tau_{m_{n}^{\prime}}}-\widetilde{X}_{\widetilde{\tau}_{m_{n}^{\prime}}}\right| \leq R\right]+\sup _{|z| \geq R} \sum_{\widetilde{z}, n} G(\widetilde{z}-z) 1\{|\widetilde{z}| \leq n\} \nu(n) .
$$

We control the first term with the help of (1.12) and (5.11) from Theorem 5.2 in the Appendix and the second term with (2.52). The claim (4.35) follows by picking $R=n^{\gamma / 4}$. This concludes the proof of Theorem 4.2.

REMARK 4.3. Note that in the situation of the example corresponding to (1.41), the $\mathbb{P}$-almost sure central limit theorem for $B^{n}$ breaks down. However if we make the additional assumption (3.1) in the directed situation, the technique of Theorem 4.2 can successfully be applied, when $d \geq 4$. This is somewhat in the same spirit as part of the results of Conlon-Song [5].

5. Appendix. If $p(x), x \in \mathbb{Z}^{d}$, is a probability measure, we write $\Sigma_{p}$ for the covariance matrix, and $p_{n}$ for the $n$-fold convolution of $p$. We set $\mu(p) \stackrel{\text { def }}{=} \sum_{x \in \mathbb{Z}^{d}} x p(x)$. The function $G$ is defined by

$$
G(x)=\sum_{i, j \geq 0} \sum_{z \in \mathbb{Z}^{d}} p_{i}(z) p_{j}(x+z) .
$$

Theorem 5.1. Assume $d \geq 4$, and let $\gamma_{1}, \gamma_{2}, \gamma_{3}>0$. There exist $K_{1}\left(d, \gamma_{1}, \gamma_{2}\right)$, and $K_{2}\left(d, \gamma_{1}, \gamma_{2}, \gamma_{3}\right)$ such that for any probability measures $p(x), x \in \mathbb{Z}^{d}$, satisfying

$$
\begin{aligned}
\sum_{x \in \mathbb{Z}^{d}} p(x) \exp \left[\gamma_{1}|x|\right] & \leq 2, \\
\Sigma_{p} & \geq \gamma_{2} I_{d}, \\
|\mu(p)| & \geq \gamma_{3},
\end{aligned}
$$

one has

$$
\begin{gathered}
\sup _{x} p_{n}(x) \leq K_{1} n^{-d / 2}, n \geq 1, \\
\sup _{x}\left(1+|x|^{(d-3) / 2}\right) G(x) \leq K_{2} .
\end{gathered}
$$

Proof. We will drop dependencies on the dimension in the notation. (5.5) follows from a concentration inequality of Esseen (Corollary to Theorem 6.2 of [7]). In fact, if $B$ is a ball with radius $1 / 2$ centered at $x \in \mathbb{Z}^{d}$, and if $P_{n}$ is the probability measure $\sum_{y} p_{n}(y) \delta_{y}$ on $\mathbb{R}^{d}$, then Esseen's inequality gives

$$
p_{n}(x)=P_{n}(B) \leq K\left(\gamma_{1}, \gamma_{2}\right) n^{-d / 2} .
$$


The constant in [7] is given explicitly in terms of the minimal eigenvalue of a truncated covariance matrix, which can easily been estimated by $\gamma_{1}, \gamma_{2}$ in our case. (Esseen's estimate is valid also without any moment conditions on $p$ ).

We now derive (5.6) from (5.5).We find $\rho\left(\gamma_{1}\right)>0$ such that

$$
p^{\lambda}(x) \stackrel{\text { def }}{=} \mathrm{e}^{\lambda \cdot x} p(x) / z(\lambda)
$$

is well defined for $\lambda \in B_{\rho}(0)$, the ball of radius $\rho$ around 0 , where $z(\lambda) \stackrel{\text { def }}{=}$ $\sum_{x} \mathrm{e}^{\lambda \cdot x} p(x)$. We can choose $\rho\left(\gamma_{1}, \gamma_{2}\right)>0$, such that $p^{\lambda}$ satisfies (5.2) and (5.3) with $\gamma_{1}, \gamma_{2}$ replaced by $\gamma_{1} / 2, \gamma_{2} / 2$ if $\lambda \in B_{\rho}(0)$. Let

$$
A_{p}(\lambda) \stackrel{\text { def }}{=} \sum_{x} x p^{\lambda}(x) .
$$

By choosing $\rho$ still smaller, if necessary, $A_{p}$ is analytic and maps $B_{\rho}(0)$ one to one on a neighborhood of $\mu(p)$ containing a ball $B_{\varepsilon}(\mu(p))$ where $\varepsilon\left(\gamma_{1}, \gamma_{2}\right)>0$. For $\xi \in B_{\varepsilon}(\mu(p))$, we find a unique $\lambda_{\xi}$ with $\left|\lambda_{\xi}\right|<\rho$, such that $\mu\left(\lambda_{\xi}\right)=\xi$. Applying this to $\xi=x / n,|x-n \mu|<n \varepsilon$, we get

$$
\begin{aligned}
p_{n}(x) & =\exp \left[-\lambda_{x / n} x+n \log z\left(\lambda_{x / n}\right)\right] p_{n}^{\lambda_{x / n}}(x) \\
& \leq K_{1}\left(\frac{\gamma_{1}}{2}, \frac{\gamma_{2}}{2}\right) n^{-d / 2} \exp [-n I(x / n)],
\end{aligned}
$$

where

$$
I(\xi) \stackrel{\text { def }}{=} \lambda_{\xi} \xi+\log z\left(\lambda_{\xi}\right)
$$

$I$ is analytic on $B_{\varepsilon}(\mu)$, is convex, and satisfies $I(\mu)=0, \nabla I(\mu)=0$, and $\nabla^{2} I(\mu)=$ $\Sigma_{p}^{-1}$. The second derivative of $I$ is positive definite in a neighborhood of $\mu(p)$ whose radius depends only on $\gamma_{1}, \gamma_{2}$, uniformly in distributions $p$, satisfying (5.2) and (5.3). It therefore follows that for some positive $\delta\left(\gamma_{1}, \gamma_{2}\right) \in(0,1]$,

$$
p_{n}(x) \leq C \varphi_{n}^{\delta}(x ; \mu(p) n)
$$

for $x / n \in B_{\varepsilon}(\mu)$, where

$$
\varphi_{n}^{\delta}(x ; m) \stackrel{\text { def }}{=} \frac{\delta^{n / 2}}{(2 \pi)^{d / 2} n^{d / 2}} \exp \left[-\delta \frac{|x-m|^{2}}{2 n}\right], n \geq 1, \varphi_{0}(x ; m) \stackrel{\text { def }}{=} \delta_{0 x},
$$

and where we use $C$ for a positive constant, depending only on the $\gamma^{\prime}$ s (and $d$ ), not necessarily the same at different occurrences. On the other hand, standard exponential estimates show that for some $\widetilde{\delta}\left(\varepsilon, \gamma_{1}\right)=\widetilde{\delta}\left(\gamma_{1}, \gamma_{2}\right)>0$, one has

$$
p_{n}(x) \leq C \exp [-\widetilde{\delta}|x-n \mu|]
$$

for $x / n \notin B_{\varepsilon}(\mu)$. Therefore we get the estimate

$$
p_{n}\left(x \leq C\left(\varphi_{n}^{\delta}(x ; n \mu)+1_{\{|x-a n| \geq \varepsilon n\}} \exp [-\widetilde{\delta}|x-n \mu|]\right),\right.
$$

uniformly in $p$ 's satisfying (5.2) and (5.3). We use that now to estimate the right-hand side of (5.1). Observe that

$$
\sum_{n \geq 0} 1_{\{|x-\mu n| \geq \varepsilon n\}} \exp [-\widetilde{\delta}|x-n \mu|] \leq C \exp \left[-\delta^{\prime}|x|\right]
$$


for some $\delta^{\prime}>0$ (depending still only on $\gamma_{1}, \gamma_{2}$ ), and it is easy to see that $\sum_{i} \varphi_{i}^{\delta}(x) \leq$ $C(1+|x|)^{-d+2}$. Therefore, the three terms in the estimate of (5.1) coming from the second summand in (5.7) can all be estimated by

$$
\sum_{z \in \mathbb{Z}^{d}}(1+|z|)^{-d+2} \exp \left[-\delta^{\prime}|x+z|\right] \leq C(1+|x|)^{-d+2},
$$

and we get

$$
G(x) \leq C \sum_{i, j \geq 1} \sum_{z} \varphi_{i}^{\delta}(z ; i \mu) \varphi_{j}^{\delta}(x+z ; j \mu)+C(1+|x|)^{-d+2} .
$$

(The summands with $i$ or $j=0$ can be incorporated into the second summand). We may also restrict the summation on the right-hand side to $j \geq i \geq 1$, catching a harmless factor 2 . We use the inequality

$$
\sum_{z} \varphi_{i}^{\delta}(z ; i \mu) \varphi_{j}^{\delta}(x+z ; j \mu) \leq C \varphi_{i+j}^{\delta}(x ;(j-i) \mu) .
$$

Postponing the proof for a moment, we can now finish the proof of (5.6) easily. Fixing $k \geq 0$, we get

$$
\begin{aligned}
\sum_{i \geq 1} \sum_{z} \varphi_{i}^{\delta}(z ; i \mu) \varphi_{i+k}^{\delta}(x+z ;(i+k) \mu) & \leq C \sum_{i \geq 1} \varphi_{2 i+k}^{\delta}(x ; k \mu) \leq C \sum_{i \geq \max (k, 1)} \varphi_{i}^{\delta}(x ; k \mu) \\
& \leq C \min \left(|x-k \mu|^{-d+2},(1+k)^{-d / 2+1}\right)
\end{aligned}
$$

and therefore

$$
G(x) \leq C \sum_{k=0}^{\infty} \min \left(|x-k \mu|^{-d+2},(1+k)^{-d / 2+1}\right) \leq C(1+|x|)^{(-d+3) / 2} .
$$

In order to prove the last inequality, first remark that (5.4) and (5.2) imply that $|\mu|$ is bounded and bounded away from 0 . Then, if $b \stackrel{\text { def }}{=} \min _{k}|x-k \mu|$, we have $\sum_{k=0}^{\infty}|x-k \mu|^{-d+2} \leq C b^{-d+3}$ by a straightforward estimate. So we can use this in case $b \geq \sqrt{|x|}$. In case $b<\sqrt{|x|}$, we take the second part in the above min in (5.10) for those $k$ where $|x-k \mu| \leq 2 \sqrt{|x|}$ getting of order $\sqrt{|x|}$ summands which are estimated by $(1+k)^{-d / 2+1} \leq C(1+|x|)^{-d / 2+1}$, and so this gives a contribution of at maximum $C(1+|x|)^{(-d+3) / 2}$. Finally, (still for $b<\sqrt{|x|}$ ), we have

$$
\sum_{k:|x-k \mu|>2 \sqrt{|x|}}|x-k \mu|^{-d+2} \leq C \sum_{j \geq \delta^{\prime \prime} \sqrt{|x|}} j^{-d+2} \leq C(1+|x|)^{(-d+3) / 2}
$$

for some $\delta^{\prime \prime}>0$. So this proves the second inequality of (5.10), and therefore the theorem. 
It remains to prove (5.9): We consider $\varphi^{\delta}$ as a function on $\mathbb{R}^{d}$, where it is just the normal density. Set $I=\left[-\frac{1}{2}, \frac{1}{2}\right]^{d}$. Then

$$
\begin{aligned}
\varphi_{i+j}^{\delta}(x ;(j-i) \mu) & =\sum_{z \in \mathbb{Z}^{d}} \int_{z+I} \varphi_{i}^{\delta}(t ; i \mu) \varphi_{j}^{\delta}(x+t ; j \mu) d t \\
& \geq \frac{\delta^{d}}{(2 \pi i)^{d / 2}(2 \pi j)^{d / 2}} \sum_{z \in \mathbb{Z}^{d}} \exp \left[-\int_{I}\left\{\frac{\delta|z+t-i \mu|^{2}}{2 i}+\frac{\delta|z+x+t-j \mu|^{2}}{2 j}\right\} d t\right],
\end{aligned}
$$

by Jensen's inequality. Note that for $t \in I$, we have $|t|^{2} \leq d / 4$, and so

$$
\begin{aligned}
& \int_{I}\left\{\frac{\delta|z+t-i \mu|^{2}}{2 i}+\frac{\delta|z+x+t-j \mu|^{2}}{2 j}\right\} d t \\
& \leq \frac{\delta|z-i \mu|^{2}}{2 i}+\frac{\delta|z+x-j \mu|^{2}}{2 j}+d / 4,
\end{aligned}
$$

if $i, j \geq 1, \delta \leq 1$, where we have used that the integral of the cross terms vanishes (by the symmetry of $I$ ). This proves the claim.

We apply this now to

$$
p(x) \stackrel{\text { def }}{=} P_{0}\left[X_{\tau_{1}}=-x \mid D^{\ell}=\infty\right]
$$

TheOREm 5.2. ( $d \geq 4$, under $(0.2),(0.9), 2<a \leq 10 \sqrt{d})$.

There exists $K>0$, depending solely on $d, \eta, \kappa$ such that

$$
\begin{gathered}
\sup _{x} p_{n}(x) \leq \frac{K}{n^{d / 2}}, \\
\sup _{x}\left(1+|x|^{(d-3) / 2}\right) G(x) \leq K
\end{gathered}
$$

Proof. We simply need to check that (5.2)-(5.4) of the previous theorem are satisfied, with $\gamma_{1}, \gamma_{2}, \gamma_{3}$ depending only on $d, \eta, \kappa$. (5.4) is evident and (5.2) follows from (1.23) and (1.24) in Sznitman [18].

It remains to prove (5.3). To this end, we consider $e_{*} \in \mathbb{Z}^{d}$, with $\left|e_{*}\right|=1$, such that

$$
e_{*} \cdot \ell=\max _{|e|=1} e \cdot \ell \geq \frac{1}{\sqrt{d}} .
$$

Then $e_{*} \cdot \ell$ and $\left(e+e_{*}\right) \cdot \ell$ for $|e|=1$ are non-negative and smaller than $a$. We consider for $|e|=1, k(e)>0$, the smallest integer such that

$$
\left(e+(k(e)+1) e_{*}\right) \cdot \ell>a .
$$

Note that

$$
\begin{aligned}
& P_{0}\left[X_{\tau_{1}}=e+(k(e)+1) e_{*} \mid D^{\ell}=\infty\right] \\
& \geq P_{0}\left[X_{1}=e_{*}, X_{2}=e_{*}+e, X_{k+2}=(k+1) e_{*}+e, 1 \leq k \leq k(e)\right] \geq \delta>0,
\end{aligned}
$$


where $\delta$ depends only on $d, \eta, \kappa$. By letting the path backtrack in the direction $e_{*}$ after reaching $e+(k(e)+1) e_{*}$, one sees that if $M$ is the smallest integer with $M\left(e_{*} \cdot \ell\right)>a$, one also has

$$
P_{0}\left[X_{\tau_{1}}=e+(k(e)+1+M) e_{*} \mid D^{\ell}=\infty\right] \geq \delta>0 .
$$

From this, we see that $p(x) \geq \delta(d, \eta, \kappa)>0$, whenever, $x$ is of the form $e+(k(e)+1) e_{*}$ or $e+(k(e)+1+M) e_{*},|e|=1$. The set of these points do not lie in a $(d-1)$-dimensional hyperplane. From this (5.3) follows.

\section{REFERENCES}

[1] N. Alon and J. Spencer and P. ERdös, The probabilistic method, John Wiley \& Sons, New York, 1992.

[2] E. Bolthausen, A note on the diffusion of directed polymers in a random environment, Comm. Math. Phys., 123 (1989), pp. 529-534.

[3] E. Bolthausen And A.S. Sznitman, Ten lectures on random media, DMV Seminar, Band 32, Birkhäuser, Basel, 2002

[4] R. Carmona and S.A. Molchanov, Parabolic Anderson problem and intermittency, Mem. Amer. Math. Soc., Providence, RI, vol. 518, 1994.

[5] J.G. Conlon And R. Song, Gaussian limit theorems for diffusion processes and an application, Stochastic Process. Appl., 81(1)(1999), pp. 103-128.

[6] R. Durrett, Probability: Theory and Examples, Wadsworth and Brooks/Cole, Pacific Grove, 1991.

[7] C.G. Esseen, On the concentration function of a sum of independent random variables, Z. Wahrscheinlichkeitstheorie verw. Geb., 9 (1968), pp. 299-308.

[8] S.M. Ethier And T.G. Kurtz, Markov processes, John Wiley \& Sons, New York, 1986.

[9] H. Kesten, A renewal theorem for random walk in a random environment, Proc. Symposia Pure Math., 31 (1977), pp. 66-77.

[10] C. Kipnis and S.R.S. Varadhan, A central limit theorem for additive functionals of reversible Markov processes and applications to simple exclusions, Comm. Math. Phys., 104 (1986), pp. 1-19.

[11] S.M. KozLOv, The method of averaging and walks in inhomogeneous environments, Russian Math. Surveys, 40(2)(1985), pp. 73-145.

[12] G.F. LAWLER, Weak convergence of a random walk in a random environment, Comm. Math. Phys., 87 (1982), pp. 81-87.

[13] S.A. Molchanov, Lectures on random media, Ecole d'Eté de Probabilités de St. Flour XXII1992, Editor P. Bernard, Lecture Notes in Mathematics, vol. 1581, Springer, 1994.

[14] S. Olla, Homogenization of diffusion processes in random fields, Ecole Polytechnique, Palaiseau, 1994.

[15] G. Papanicolaou and S.R.S. Varadhan, Diffusion with random coefficients. Statistics and probability: essays in honor of C.R. Rao, G. Kallianpur, P.R. Krishnajah, J.K. Gosh, eds., North Holland, Amsterdam, pp. 547-552, 1982.

[16] Ya.G. SinAI, A remark concerning random walk with random potentials, Fund. Math., 147 (1995), pp. 173-180.

[17] F. Spitzer, Principles of random walk, Springer, Berlin, second edition, 1976.

[18] A.S. Sznitman, Slowdown estimates and central limit theorem for random walks in random environment, J. Eur. Math. Soc., 2 (2000), pp. 93-143.

[19] A.S. Sznitman, An effective criterion for ballistic behavior of random walks in random environment, Probab. Theory Relat. Fields, 122(4)(2002), pp. 509-544.

[20] A.S. Sznitman And M.P.W. Zerner, A law of large numbers for random walks in random environment, Ann. Probab., 27(4)(1999), pp. 1851-1869. 
E. BOLTHAUSEN AND A.-S. SZNITMAN 